\title{
A Unified Approach to Singularly Perturbed Quasilinear Schrödinger Equations
}

\author{
Daniele Cassani, Youjun Wang and Jianjun Zhang
}

\begin{abstract}
In this paper we present a unified approach to investigate existence and concentration of positive solutions for the following class of quasilinear Schrödinger equations,$$
-\varepsilon^{2} \Delta u+V(x) u \mp \varepsilon^{2+\gamma} u \Delta u^{2}=h(u), \quad x \in \mathbb{R}^{N},
$$

where $N \geqslant 3, \varepsilon>0, V(x)$ is a positive external potential, $h$ is a real function with subcritical or critical growth. The problem is quite sensitive to the sign changing of the quasilinear term as well as to the presence of the parameter $\gamma>0$. Nevertheless, by means of perturbation type techniques, we establish the existence of a positive solution $u_{\varepsilon, \gamma}$ concentrating, as $\varepsilon \rightarrow 0$, around minima points of the potential.
\end{abstract}

Mathematics Subject Classification (2010). 35A15, 35J20, 35J62.

Keywords. Semiclassical states, critical growth, quasilinear Schrödinger equations, variational methods.

\section{Introduction}

In this paper, we consider the following class of quasilinear Schrödinger equations,

$$
-\varepsilon^{2} \Delta u+V(x) u \mp \varepsilon^{2+\gamma} u \Delta u^{2}=h(u), \quad x \in \mathbb{R}^{N},
$$

where $N \geqslant 3, \varepsilon, \gamma>0, V$ is the external Schrödinger potential, $h$ is a real function. Solutions of (1.1) are related to standing wave solutions for the time-dependent quasilinear Schrödinger equations

$$
i \varepsilon z_{t}=-\varepsilon^{2} \Delta z+W(x) z+\kappa z \Delta|z|^{2}-f\left(|z|^{2}\right) z, \quad x \in \mathbb{R}^{N}
$$

where $z: \mathbb{R} \times \mathbb{R}^{N} \rightarrow \mathbb{C}, W: \mathbb{R}^{N} \rightarrow \mathbb{R}$ is a given potential, $f: \mathbb{R} \rightarrow \mathbb{R}$ is a real function, $\varepsilon>0$, and $\kappa$ is a parameter. An equation of type (1.2) appears in

Corresponding author: Daniele.Cassani@uninsubria.it

The second-named author is supported by the Fundamental Research Funds for the Central Universities (No.2018MS59) and Natural Science Foundation of Guangdong (No.2018A0303130196).

The third-named author was supported by NSFC(No.11871123). 
the literature in the context of plasma physics and the continuum limit of discrete molecular structures. In particular, $\kappa$ turns out to be small and negative when (1.2) represents the weak nonlocal limit of some general nonlocal models, whereas $\kappa$ has no prescribed sign in plasma physics, see for instance $[6,7,20,26,28]$ for more details on the Physics involved.

A well-known standard tool in the investigation of the elliptic side of Schrödinger equations, is the ansatz $z(t, x)=\exp (-i E t / \varepsilon) u(x)$ where $E \in \mathbb{R}$ and $u$ is a real function, by which equation (1.2) turns into the following quasilinear elliptic equation,

$$
-\varepsilon^{2} \Delta u+V(x) u-\kappa u \Delta u^{2}=h(u), \quad x \in \mathbb{R}^{N},
$$

where $V(x)=W(x)-E$ and $h(u)=f\left(|u|^{2}\right) u$.

On the one hand, the study of the existence of ground states (minimal energy solutions) and bound states (finite energy) solutions to (1.3) with $\varepsilon=1$ has received considerable attention in recent years, see [12, 23, 24, 27, 30, 34] for arbitrary fixed $\kappa>0$ and $[4,32]$ for $\kappa<0$ and $|\kappa|$ small. On the other hand, al lot of work has been done on the existence of semi-classical states for (1.3), namely $\kappa=\varepsilon^{2}$ and $\varepsilon \rightarrow 0^{+}$. Assume $V: \mathbb{R}^{N} \rightarrow \mathbb{R}$ is Hölder continuous and satisfies the following condition $(V)$ :

$$
0<V_{0}<\inf _{x \in \mathbb{R}^{N}} V(x)
$$

and there is a bounded open set $\mathcal{O}$ such that

$$
0<m:=\inf _{x \in \mathcal{O}} V(x)<\min _{x \in \partial \mathcal{O}} V(x) .
$$

With the assumption $(V)$, the existence of a localized solution concentrating around $\mathcal{M}:=\{x \in \mathcal{O}: V(x)=m\}$ has been studied under various conditions on the nonlinearity $h$. When $N \geqslant 3$, we refer to $[11,16,36]$ for the subcritical case and $[18,33]$ for the critical case. The case of dimension $N=2$ has been addressed in [13,14]. Recently, S. Adachi et al. in [1-3] have studied asymptotic properties of the ground state of the quasilinear Schrödinger equation

$$
-\Delta u+\lambda u-\kappa u \Delta u^{2}=|u|^{p-1} u, \quad x \in \mathbb{R}^{N} .
$$

As $\kappa \rightarrow 0^{+}$, they have proved that the ground state $u_{\kappa}$ convergences to a ground state solution of a limit equation for $2<p<2^{*}$ whence it blows up, in a suitable sense, for $p>2^{*}$.

Therefore, it is a legitimate and somewhat interesting question what happens when the two parameters $\varepsilon$ and $\kappa$ in (1.3) tend to zero at different speed rates.

The purpose of this paper is to present a unified approach to study the asymptotic properties of the solutions to (1.3) for $\kappa=\mp \varepsilon^{2+\gamma}$ with $\gamma>0$ and $\varepsilon \rightarrow 0^{+}$. Let us stress the fact that both signs for $\kappa$, which is assumed to be small in absolute value, turn out to be physically relevant as developed in [31]. We will show that beyond the expected concentration phenomenon, the limit equations for the case $\gamma>0$ and $\gamma=0$ turn out to be different when $\kappa=\varepsilon^{2+\gamma}$. Moreover, to the best of our knowledge, no result is known in the case $\kappa=-\varepsilon^{2+\gamma}$. 
Observe that (1.1) is the Euler-Lagrange equation associated to the energy functional

$$
I_{\varepsilon, \gamma}(u)=\frac{1}{2} \int_{\mathbb{R}^{N}}\left[\left(\varepsilon^{2}+\kappa u^{2}\right)|\nabla u|^{2}+V(x) u^{2}\right] d x-\int_{\mathbb{R}^{N}} H(u) d x,
$$

where $H(t)=\int_{0}^{t} h(s) d s$. From the variational point of view, the first difficulty to handle is to find a proper function space setting where (1.4) is well defined. For $\kappa=\varepsilon^{2+\gamma}$, this difficulty can be overcome by minimization on spheres (mass constraint) or on the Nehari manifold, see [22,24]. Another important tool for this type of equations is to perform a suitable change of variable [23,29] which reduces (1.1) to a semilinear elliptic equation for which variational methods are available, see [12]. Unfortunately, the methods which work in the case $\kappa=\varepsilon^{2+\gamma}$ can not be directly adapted to deal with the case $\kappa=-\varepsilon^{2+\gamma}$. Indeed, here the principle part of the corresponding energy functional does change sign, see $[4,32]$.

As we look for positive solutions, let us assume $h(t)=0$ for $t \leqslant 0$ and we require $h \in C\left(\mathbb{R}^{+}, \mathbb{R}\right)$ to enjoy the following conditions, which were introduced by Byeon and Jeanjean in [10], see also [9]:

$\left(h_{1}\right) \lim _{t \rightarrow 0} \frac{h(t)}{t}=0$;

$\left(h_{2}\right) \lim _{t \rightarrow+\infty} \frac{h(t)}{t^{p}}=0, p \in\left(1,2^{*}-1\right)$;

$\left(h_{3}\right)$ there exits $T>0$ such that $\frac{1}{2} m T^{2}<H(T)$.

Our main result is the following:

Theorem 1.1 (Subcritical case). Let $\gamma>0$ and assume that $(V)$ and $\left(h_{1}\right)-\left(h_{3}\right)$ hold. Then, for sufficiently small $\varepsilon>0$, there exists a positive solution $u_{\varepsilon, \gamma}$ of (1.1) satisfying the following:

(1) There exists a local maximum point $x_{\varepsilon}$ of $u_{\varepsilon, \gamma}$ such that $\lim _{\varepsilon \rightarrow 0} \operatorname{dist}\left(x_{\varepsilon}, \mathcal{M}\right)=0$ and $u_{\varepsilon, \gamma}\left(\varepsilon \cdot-\varepsilon x_{\varepsilon}\right)$ converges locally uniformly to a positive ground state of

$$
-\Delta u+m u=h(u)
$$

(2) There exist $C, c>0$ such that

$$
u_{\varepsilon, \gamma}(x) \leqslant C \exp \left(-\frac{c}{\varepsilon}\left|x-x_{\varepsilon}\right|\right)
$$

for all $x \in \mathbb{R}^{N}$.

Critical case. Assume in place of $\left(h_{2}\right)$ the following critical growth,

$$
\lim _{t \rightarrow+\infty} \frac{h(t)}{t^{2^{*}-1}}=1
$$


The above results extend to cover the critical case provided we further assume

$$
\left(h_{5}\right) \begin{cases}\lim _{t \rightarrow+\infty} \frac{H(t)-\frac{1}{2^{*}} t^{2^{*}}}{t^{4}}=+\infty, & \text { if } N=3 ; \\ \lim _{t \rightarrow+\infty} \frac{H(t)-\frac{1}{2^{*}} t^{2^{*}}}{t^{2} \ln t}=+\infty, & \text { if } N=4 ; \\ \lim _{t \rightarrow+\infty} \frac{H(t)-\frac{1}{2^{*}} t^{2^{*}}}{t^{2}}=+\infty, & \text { if } N \geq 5 .\end{cases}
$$

In [16], similar results to those of Theorem 1.1 are obtained for the equation (1.3) with $\gamma=0$, namely $\kappa=\varepsilon^{2}$ and $p \in\left(1,2\left(2^{*}\right)-1\right)$ in $\left(h_{2}\right)$. The limit equation for $\kappa=\varepsilon^{2}$ being

$$
-\Delta u+m u-u \Delta u^{2}=h(u) .
$$

In our case, where $\kappa=\varepsilon^{2+\gamma}$ with $\gamma>0$, the situation is different and we can not cover the range $p \in\left(2^{*}-1,2\left(2^{*}\right)-1\right)$ as the solution can blow up. Closely related problems were considered in [4], where the authors study the following equation,

$$
-\Delta u+V(x) u+\alpha u \Delta u^{2}=|u|^{p-1} u, \quad x \in \mathbb{R}^{N}
$$

and assuming $V$ is a trapping potential, they have proved the existence of a positive solution for $2<p<2^{*}-1$ and $\alpha>0$ small enough. Moreover, in [32] the authors prove that if $V \in C^{1}\left(\mathbb{R}^{N},[0,+\infty)\right)$ is such that $x \cdot \nabla V(x)+2 V(x) \geqslant 0$ for all $x \in \mathbb{R}^{N}$, then the equation (1.6) has only trivial $C^{2}$ solutions for any $p \geqslant 2^{*}-1$ and any $\alpha>0$.

Finally, let us point out that difficulties in (1.3) with $\kappa=-\varepsilon^{2}$ are due to the fact that it is still an open problem if there exists a solution to equation (1.6) for all $\alpha>0$.

The outline of the paper is as follows: in Section 2, we modify (1.1) by introducing an auxiliary function. In Section 3, we prove the existence of a positive solution for the modified problem. Then, Sections 4 and 5 are devoted to prove Theorem 1.1 respectively in the subcritical and critical case where we use a truncation approach.

Notation. Without loss of generality, we may assume $0 \in \mathcal{M}$. For any set $\Omega \subset \mathbb{R}^{N}$ and $\varepsilon, \delta>0$, we define $\Omega_{\varepsilon}:=\left\{x \in \mathbb{R}^{N}: \varepsilon x \in \Omega\right\}$ and $\Omega^{\delta}:=\left\{x \in \mathbb{R}^{N}: \operatorname{dist}(x, \Omega) \leqslant \delta\right\}$. Let

$$
E_{\varepsilon}:=\left\{v \in H^{1}\left(\mathbb{R}^{N}\right): \int_{\mathbb{R}^{N}} V(\varepsilon x) v^{2} d x<\infty\right\}
$$

the Hilbert space endowed with the norm $\|v\|_{\varepsilon}:=\sqrt{\int_{\mathbb{R}^{N}}\left(|\nabla v|^{2}+V(\varepsilon x) v^{2}\right) d x}$. We denote by $C$ a positive constants whose exact value may change from line to line without affecting the overall result.

\section{An auxiliary problem}

Let $w(x)=u(\varepsilon x)$, equation (1.1) is equivalent to

$$
-\Delta w+V_{\varepsilon}(x) w \mp \varepsilon^{\gamma} w \Delta w^{2}=h(w), \quad x \in \mathbb{R}^{N},
$$

where $V_{\varepsilon}(x)=V(\varepsilon x)$. 
Note that (2.1) formally is the Euler-Lagrange equation associated to the energy functional

$$
I_{\varepsilon, \gamma}(w)=\frac{1}{2} \int_{\mathbb{R}^{N}}\left[\left(1 \pm \varepsilon^{\gamma} w^{2}\right)|\nabla w|^{2}+V_{\varepsilon}(x) w^{2}\right] d x-\int_{\mathbb{R}^{N}} H(w) d x .
$$

However, classical variational methods can not be directly applied since $I_{\varepsilon, \gamma}$ is not well defined on the Hilbert space $E_{\varepsilon}$ because of the presence of four homogeneous term $w^{2}|\nabla w|^{2}$.

Define an auxiliary function $g_{\varepsilon, \gamma, \pm}(t): \mathbb{R} \rightarrow \mathbb{R}^{+}$as follows:

$$
g_{\varepsilon, \gamma, \pm}(t)=\sqrt{1 \pm \varepsilon^{\gamma} t^{2} \eta(t)}
$$

where $\eta(t) \in C_{0}^{\infty}(\mathbb{R},[0,1])$ is a cut-off function such that $\eta(t)=\eta(-t), \eta(t)=1$ for $0 \leqslant t<\sqrt[4]{1 / \varepsilon^{\gamma}}$ and $\eta(t)=0$ for $t \geqslant 2 \sqrt[4]{1 / \varepsilon^{\gamma}}$. Moreover, there exists some $C>0$ independent of $\varepsilon$ and $\gamma$ such that $-C \sqrt{\eta(t)} \leqslant \eta^{\prime}(t) t \leqslant 0$, for all $t \in \mathbb{R}$.

Remark 2.1. The cut-off function $\eta(t)$ can be constructed as follows. Let

$$
\zeta(t)=\frac{1}{1+e^{\frac{1}{t}-\frac{1}{1-t}}}
$$

for $0<t<1, \zeta(t)=1$ for $t \geqslant 1$ and $\zeta(t)=0$ for $t \leqslant 0$. For $t \in \mathbb{R}^{+}$let $\rho(t)=\zeta^{2}\left[2-\sqrt[4]{\varepsilon^{\gamma}} t\right]$. Then, the function $\eta(t)=\rho(t)$ for $t \geqslant 0$ and $\eta(t)=\rho(-t)$ for $t<0$, enjoys the required properties.

Let

$$
G_{\varepsilon, \gamma, \pm}(t)=\int_{0}^{t} g_{\varepsilon, \gamma, \pm}(s) d s
$$

then $G_{\varepsilon, \gamma, \pm}(t)$ is an odd and smooth function as well as the inverse function $G_{\varepsilon, \gamma, \pm}^{-1}(t)$. For any fixed $\gamma>0$ and small $\varepsilon>0$, next we establish a few properties of $G_{\varepsilon, \gamma, \pm}^{-1}(t)$.

Lemma 2.2. The following properties hold:

(i) $\lim _{t \rightarrow 0} \frac{G_{\varepsilon, \gamma, \pm}^{-1}(t)}{t}=1$;

(ii) $\lim _{t \rightarrow+\infty} \frac{G_{\varepsilon, \gamma, \pm}^{-1}(t)}{t}=1$;

(iii) $\frac{1}{\sqrt{1+4 \varepsilon^{\frac{\gamma}{2}}}}|t| \leqslant\left|G_{\varepsilon, \gamma,+}^{-1}(t)\right| \leqslant|t|$ and $|t| \leqslant\left|G_{\varepsilon, \gamma,-}^{-1}(t)\right| \leqslant \frac{1}{\sqrt{1-4 \varepsilon^{\frac{\gamma}{2}}}}|t|$ for all $t \in \mathbb{R}$;

(iv) there exists $C>0$ such that $\left|G_{\varepsilon, \gamma, \pm}^{-1}(t)-t\right| \leqslant C \sqrt{\varepsilon^{\gamma}}|t|$;

(v) $\left|G_{\varepsilon, \gamma, \pm}^{-1}(t)\right|^{2}$ is convex in $t$ for small $\varepsilon>0$.

Proof. (i) and (ii) are consequences of L'Hôspital's rule:

$$
\lim _{t \rightarrow 0(+\infty)} \frac{G_{\varepsilon, \gamma, \pm}^{-1}(t)}{t}=\lim _{t \rightarrow 0(+\infty)} \frac{1}{g_{\varepsilon, \gamma, \pm}\left(G_{\varepsilon, \gamma, \pm}^{-1}(t)\right)}=1 .
$$

By definition of $g_{\varepsilon, \gamma, \pm}(t)$ and $G_{\varepsilon, \gamma, \pm}(t)$, (iii) follows.

Let us prove (iv): for $t>0$ we have

$$
\left|G_{\varepsilon, \gamma, \pm}(t)-t\right| \leqslant \int_{0}^{t}\left|g_{\varepsilon, \gamma, \pm}(s)-1\right| d s \leqslant \varepsilon^{\gamma} \int_{0}^{t} s^{2} \eta(s) d s \leqslant 4 \sqrt{\varepsilon^{\gamma}} t
$$


From (iii), we have $\left|G_{\varepsilon, \gamma, \pm}^{-1}(t)-t\right| \leqslant 4 \sqrt{\varepsilon^{\gamma}}\left|G_{\varepsilon, \gamma, \pm}^{-1}(t)\right| \leqslant C \sqrt{\varepsilon^{\gamma}}|t|$.

Finally let $T_{ \pm}(t):=\left|G_{\varepsilon, \gamma, \pm}^{-1}(t)\right|^{2}$. Then, for small $\varepsilon>0$, by (iii) we have

$$
\begin{aligned}
\frac{1}{2} g^{4}\left(G_{\varepsilon, \gamma,+}^{-1}(t)\right) T_{+}^{\prime \prime}\left(G_{\varepsilon, \gamma,+}^{-1}(t)\right)= & 1-\varepsilon^{\gamma}\left|G_{\varepsilon, \gamma,+}^{-1}(t)\right|^{2} \eta\left(G_{\varepsilon, \gamma,+}^{-1}(t)\right) \\
& -\varepsilon^{\gamma}\left|G_{\varepsilon, \gamma,+}^{-1}(t)\right|^{2} \eta^{\prime}\left(G_{\varepsilon, \gamma,+}^{-1}(t)\right) G_{\varepsilon, \gamma,+}^{-1}(t)>0
\end{aligned}
$$

and

$$
\begin{aligned}
\frac{1}{2} g^{4}\left(G_{\varepsilon, \gamma,-}^{-1}(t)\right) T^{\prime \prime}\left(G_{\varepsilon, \gamma,-}^{-1}(t)\right)= & 1+\varepsilon^{\gamma}\left|G_{\varepsilon, \gamma,-}^{-1}(t)\right|^{2} \eta\left(G_{\varepsilon, \gamma,-}^{-1}(t)\right) \\
& +\varepsilon^{\gamma}\left|G_{\varepsilon, \gamma,-}^{-1}(t)\right|^{2} \eta^{\prime}\left(G_{\varepsilon, \gamma,-}^{-1}(t)\right) G_{\varepsilon, \gamma,-}^{-1}(t)>0,
\end{aligned}
$$

which imply (v).

Next we consider the following modified quasilinear Schrödinger equation,

$$
-\operatorname{div}\left(g_{\varepsilon, \gamma, \pm}^{2}(w) \nabla w\right)+g_{\varepsilon, \gamma, \pm}(w) g_{\varepsilon, \gamma, \pm}^{\prime}(w)|\nabla u|^{2}+V_{\varepsilon}(x) w=h(w) .
$$

Direct calculations show that (2.4) turns into (2.1) when $g_{\varepsilon, \gamma, \pm}(t)=\sqrt{1 \pm \varepsilon^{\gamma} t^{2}}$ and the energy functional related to (2.4) is given by

$$
\bar{I}_{\varepsilon, \gamma, \pm}(w)=\frac{1}{2} \int_{\mathbb{R}^{N}}\left[g_{\varepsilon, \gamma, \pm}^{2}(w)|\nabla w|^{2}+V_{\varepsilon}(x) w^{2}\right] d x-\int_{\mathbb{R}^{N}} H(w) d x
$$

which is well defined on $E_{\varepsilon}$, though it is still non-smooth. As in [29], we exploit the change of variable $w=G_{\varepsilon, \gamma, \pm}^{-1}(v)$ to replace the functional $\bar{I}_{\varepsilon, \gamma, \pm}$ by the following smooth functional:

$$
P_{\varepsilon, \gamma, \pm}(v)=\frac{1}{2} \int_{\mathbb{R}^{N}}\left(|\nabla v|^{2}+V_{\varepsilon}(x)\left|G_{\varepsilon, \gamma, \pm}^{-1}(v)\right|^{2}\right) d x-\int_{\mathbb{R}^{N}} H\left(G_{\varepsilon, \gamma, \pm}^{-1}(v)\right) d x .
$$

Indeed, under conditions $\left(h_{1}\right)-\left(h_{3}\right)$ and (i)-(ii) of Lemma 2.2, it is standard to check that $P_{\varepsilon, \gamma, \pm} \in C^{1}\left(E_{\varepsilon}, \mathbb{R}\right)$.

The Euler-Lagrange equation associated to $P_{\varepsilon, \gamma, \pm}$ is

$$
-\Delta v+V_{\varepsilon}(x) \frac{G_{\varepsilon, \gamma, \pm}^{-1}(v)}{g_{\varepsilon, \gamma, \pm}\left(G_{\varepsilon, \gamma, \pm}^{-1}(v)\right)}=\frac{h\left(G_{\varepsilon, \gamma, \pm}^{-1}(v)\right)}{g_{\varepsilon, \gamma, \pm}\left(G_{\varepsilon, \gamma, \pm}^{-1}(v)\right)}, \quad x \in \mathbb{R}^{N} .
$$

Now notice that if $v \in E_{\varepsilon}$ is a critical point of $P_{\varepsilon, \gamma, \pm}(v)$, then it satisfies for all $\phi \in E_{\varepsilon}$,

$$
\int_{\mathbb{R}^{N}}\left[\nabla v \nabla \phi+V_{\varepsilon}(x) \frac{G_{\varepsilon, \gamma, \pm}^{-1}(v)}{g_{\varepsilon, \gamma, \pm}\left(G_{\varepsilon, \gamma, \pm}^{-1}(v)\right)} \phi\right] d x-\int_{\mathbb{R}^{N}} \frac{h\left(G_{\varepsilon, \gamma, \pm}^{-1}(v)\right)}{g_{\varepsilon, \gamma, \pm}\left(G_{\varepsilon, \gamma, \pm}^{-1}(v)\right)} \phi d x=0 .
$$

Let $w=G_{\varepsilon, \gamma, \pm}^{-1}(v) \in E_{\varepsilon}, \varphi \in C_{0}^{\infty}\left(\mathbb{R}^{N}\right)$, then $\phi:=\varphi g_{\varepsilon, \gamma, \pm}\left(G_{\varepsilon, \gamma, \pm}^{-1}(v)\right) \in E_{\varepsilon}$ and

$$
\int_{\mathbb{R}^{N}}\left[\nabla w \nabla \varphi+g_{\varepsilon, \gamma, \pm}(w) g_{\varepsilon, \gamma, \pm}^{\prime}(w)|\nabla w|^{2} \varphi+V_{\varepsilon}(x) w \varphi\right] d x-\int_{\mathbb{R}^{N}} h(w) \varphi d x=0,
$$

which implies that $w$ is a weak solution of (2.4).

Define the functional

$$
\Gamma_{\varepsilon, \gamma, \pm}(v)=P_{\varepsilon, \gamma, \pm}(v)+Q_{\varepsilon}(v)
$$


where

$$
Q_{\varepsilon}(v)=\left(\int_{\mathbb{R}^{N}} \chi_{\varepsilon} v^{2} d x-1\right)_{+}^{2}
$$

with $\chi_{\varepsilon}(x)=0$ for $x \in \mathcal{O}_{\varepsilon}$ and $\chi_{\varepsilon}(x)=\varepsilon^{-1}$ for $x \notin \mathcal{O}_{\varepsilon}$. The functional $Q_{\varepsilon}$ will have a penalization effect which forces concentration phenomena to occur inside $\mathcal{O}$. This type of penalization was first introduced in [8]. Clearly, $\Gamma_{\varepsilon, \gamma, \pm} \in C^{1}\left(E_{\varepsilon}, \mathbb{R}\right)$.

\section{Existence of solutions for the modified problem}

Let us recall some well known results about the limit equation (1.5) under conditions $\left(h_{1}\right)-\left(h_{3}\right)$. The energy functional is defined by

$$
L_{m}(v)=\frac{1}{2} \int_{\mathbb{R}^{N}}\left(|\nabla v|^{2}+m v^{2}\right) d x-\int_{\mathbb{R}^{N}} H(v) d x .
$$

In [5], Berestycki and Lions proved that there exists a ground state (least energy solution) of (1.5) and each solution $v$ of (1.5) satisfies Pohozaev's identity

$$
\frac{N-2}{2 N} \int_{\mathbb{R}^{N}}|\nabla v|^{2} d x+\frac{1}{2} m \int_{\mathbb{R}^{N}} v^{2} d x-\int_{\mathbb{R}^{N}} H(v) d x=0 .
$$

Let $S_{m}$ be the set of ground states $U$ of (1.5) satisfying $U(0)=\max _{x \in \mathbb{R}^{N}} U(x)$. Then, $S_{m}$ is compact and

$$
|U(x)|+|\nabla U(x)| \leqslant C \exp (-c|x|), \quad x \in \mathbb{R}^{N},
$$

for some $C, c>0$ independent of $U$, see Proposition 1 in [10]. In [19], Jeanjean and Tanaka proved that the ground state has a mountain pass characterization, i.e.,

$$
L_{m}(U)=C_{m}=\inf _{\eta \in \Phi} \max _{t \in[0,1]} L_{m}(\eta(t)),
$$

where $\Phi=\left\{\eta \in C\left([0,1], H^{1}\left(\mathbb{R}^{N}\right)\right): \eta(0)=0, L_{m}(\eta(1))<0\right\}$.

Now set $U_{t}(x)=U\left(\frac{x}{t}\right)$ for $t>0$. By (3.1), for any $t>0$, we get

$$
L_{m}\left(U_{t}\right)=\left(\frac{t^{N-2}}{2}-\frac{t^{N}}{2^{*}}\right) \int_{\mathbb{R}^{N}}|\nabla U|^{2} d x .
$$

So, there exists $t_{0}>1$ such that $L_{m}\left(U_{t}\right)<-2$ for all $t \geqslant t_{0}$.

Choose a positive number $\beta<\frac{\operatorname{dist}\left(\mathcal{M}, \mathbb{R}^{N} \backslash \mathcal{O}\right)}{100}$ and a cut-off funtion $\varphi(x) \in$ $C_{0}^{\infty}\left(\mathbb{R}^{N},[0,1]\right)$ such that $\varphi(x)=1$ for $|x| \leqslant \beta$ and $\varphi(x)=0$ for $|x| \geqslant 2 \beta$. We define $\varphi_{\varepsilon}(x):=\varphi(\varepsilon x)$ and $U_{\varepsilon}^{y}(x):=\varphi_{\varepsilon}\left(x-\frac{y}{\varepsilon}\right) U\left(x-\frac{y}{\varepsilon}\right)$ for each $y \in \mathcal{M}^{\beta}$, $U \in S_{m}$. For sufficiently small $\varepsilon>0$, we will find a solution near the set $X_{\varepsilon}:=\left\{U_{\varepsilon}^{y}(x): y \in \mathcal{M}^{\beta}, U \in S_{m}\right\}$.

Let $W_{\varepsilon, t}(x)=\varphi_{\varepsilon}(x) U_{t}(x)$. Note that for fixed $x \in \mathbb{R}^{N}, W_{\varepsilon, t}(x) \rightarrow 0$ as $t \rightarrow 0$. Thus, we set $W_{\varepsilon, 0}(x)=0$.

Lemma 3.1. $\lim _{\varepsilon \rightarrow 0} \max _{t \in\left(0, t_{0}\right]}\left|\Gamma_{\varepsilon, \gamma, \pm}\left(W_{\varepsilon, t}\right)-L_{m}\left(U_{t}\right)\right|=0$. 
Proof. Since $0 \in \mathcal{M}$, we get $\operatorname{supp}\left(W_{\varepsilon, t}(x)\right) \subset\left\{x \in \mathbb{R}^{N}:|\varepsilon x| \leqslant 2 \beta\right\} \subset\left(\mathcal{M}^{2 \beta}\right)_{\varepsilon} \subset \mathcal{O}_{\varepsilon}$. Thus, $Q_{\varepsilon}\left(W_{\varepsilon, t}(x)\right)=0$. For $t \in\left(0, t_{0}\right]$, we have

$$
\begin{aligned}
& \Gamma_{\varepsilon, \gamma, \pm}\left(W_{\varepsilon, t}\right)-L_{m}\left(U_{t}\right)=\frac{1}{2} \int_{\mathbb{R}^{N}}\left(\left|\nabla W_{\varepsilon, t}\right|^{2}-\left|\nabla U_{t}\right|^{2}\right) d x \\
& +\frac{1}{2} \int_{\mathbb{R}^{N}}\left(V_{\varepsilon}\left|G_{\varepsilon, \gamma, \pm}^{-1}\left(W_{\varepsilon, t}\right)\right|^{2}-m U_{t}^{2}\right) d x+\int_{\mathbb{R}^{N}}\left[H\left(U_{t}\right)-H\left(G_{\varepsilon, \gamma, \pm}^{-1}\left(W_{\varepsilon, t}\right)\right)\right] d x .
\end{aligned}
$$

Next we prove that the three integrals on the right-hand side of (3.3) tend to zero as $\varepsilon \rightarrow 0$. By (3.2) we have, as $\varepsilon \rightarrow 0$,

$$
\left.\int_{\mathbb{R}^{N}}|| \nabla W_{\varepsilon, t}\right|^{2}-\left|\nabla U_{t}\right|^{2} \mid d x \leqslant C \int_{\mathbb{R}^{N}}\left[C \varepsilon+\left(1-\varphi_{\varepsilon}^{2}\right)\right] e^{-c|x| / t_{0}} d x \rightarrow 0 .
$$

By Lemma 2.2-(iv) we have

$$
\left.\int_{\mathbb{R}^{N}}|| G_{\varepsilon, \gamma, \pm}^{-1}\left(U_{t}\right)\right|^{2}-\left|U_{t}\right|^{2} \mid d x \leqslant C \sqrt{\varepsilon^{\gamma}} \int_{\mathbb{R}^{N}} U^{2} d x \leqslant C \sqrt{\varepsilon^{\gamma}} .
$$

Thus, by (3.5) we have

$$
\begin{aligned}
& \left.\int_{\mathbb{R}^{N}}\left|V_{\varepsilon}\right| G_{\varepsilon, \gamma, \pm}^{-1}\left(W_{\varepsilon, t}\right)\right|^{2}-m\left|U_{t}\right|^{2} \mid d x \\
& \leqslant C \int_{\mathbb{R}^{N}}\left|V_{\varepsilon}-m\right|\left|U_{t}\right|^{2} d x+C m \int_{\mathbb{R}^{N}}\left|\xi_{\varepsilon, t}\right|\left|\left(\varphi_{\varepsilon}-1\right) U_{t}\right| d x+C \sqrt{\varepsilon^{\gamma}} \\
& \leqslant C \int_{\mathbb{R}^{N}}\left|V_{\varepsilon}-m\right| e^{-2 c|x| / t_{0}} d x+C \int_{\mathbb{R}^{N}}\left|\varphi_{\varepsilon}-1\right| e^{-c|x| / t_{0}} d x+C \sqrt{\varepsilon^{\gamma}} \rightarrow 0,
\end{aligned}
$$

as $\varepsilon \rightarrow 0$, where $\xi_{\varepsilon, t}=\tau W_{\varepsilon, t}+(1-\tau) U_{t}, 0<\tau<1,\left|\xi_{\varepsilon, t}\right| \leqslant C$.

By $\left(h_{1}\right)-\left(h_{2}\right)$ we have

$$
\left.\int_{\mathbb{R}^{N}}\left|H\left(U_{t}\right)-H\left(G_{\varepsilon, \gamma, \pm}^{-1}\left(U_{t}\right)\right)\right|\right] d x \leqslant C \int_{\mathbb{R}^{N}}\left(|U|+|U|^{p}\right)\left|U-G_{\varepsilon, \gamma, \pm}^{-1}(U)\right| d x \leqslant C \sqrt{\varepsilon^{\gamma}} .
$$

From (3.7) we get

$$
\begin{aligned}
& \int_{\mathbb{R}^{N}}\left|H\left(U_{t}\right)-H\left(G_{\varepsilon, \gamma, \pm}^{-1}\left(W_{\varepsilon, t}\right)\right)\right| d x \\
& \leqslant \int_{\mathbb{R}^{N}}\left|H\left(G_{\varepsilon, \gamma, \pm}^{-1}\left(U_{t}\right)\right)-H\left(G_{\varepsilon, \gamma, \pm}^{-1}\left(W_{\varepsilon, t}\right)\right)\right| d x+\int_{\mathbb{R}^{N}}\left|H\left(U_{t}\right)-H\left(G_{\varepsilon, \gamma, \pm}^{-1}\left(U_{t}\right)\right)\right| d x \\
& \leqslant \int_{\mathbb{R}^{N}} \frac{h\left(G_{\varepsilon, \gamma, \pm}^{-1}(\eta)\right)}{g_{\varepsilon, \gamma, \pm}\left(G_{\varepsilon, \gamma, \pm}^{-1}(\eta)\right)}\left|U_{t}-W_{\varepsilon, t}\right| d x+C \sqrt{\varepsilon^{\gamma}} \\
& \leqslant C \int_{\mathbb{R}^{N}}\left[U_{t}+W_{\varepsilon, t}+U_{t}^{p}+W_{\varepsilon, t}^{p}\right]\left|U_{t}-W_{\varepsilon, t}\right| d x+C \sqrt{\varepsilon^{\gamma}} \\
& \leqslant C \int_{\mathbb{R}^{N}}\left|\varphi_{\varepsilon}-1\right| \exp \left(-c|x| / t_{0}\right) d x+C \sqrt{\varepsilon^{\gamma}} \rightarrow 0, \text { as } \varepsilon \rightarrow 0 .
\end{aligned}
$$

Combining (3.3)-(3.8) we obtain the result. 
From Lemma 3.1, there exists $\varepsilon_{0}>0$, such that for $\varepsilon \in\left(0, \varepsilon_{0}\right)$,

$$
\Gamma_{\varepsilon, \gamma, \pm}\left(W_{\varepsilon, t_{0}}\right) \leqslant L_{m}\left(U_{t_{0}}\right)+1<-1 .
$$

Fix $\varepsilon \in\left(0, \varepsilon_{0}\right)$ and define the minimax level

$$
C_{\varepsilon, \gamma, \pm}=\inf _{\eta_{\varepsilon} \in \Phi_{\varepsilon}} \max _{s \in[0,1]} \Gamma_{\varepsilon, \gamma, \pm}\left(\eta_{\varepsilon}(s)\right),
$$

where $\Phi_{\varepsilon}=\left\{\eta_{\varepsilon} \in C\left([0,1], E_{\varepsilon}\right): \eta_{\varepsilon}(0)=0, \eta_{\varepsilon}(1)=W_{\varepsilon, t_{0}}\right\}$.

Lemma 3.2. $\lim _{\varepsilon \rightarrow 0} C_{\varepsilon, \gamma, \pm}=C_{m}$.

Proof. Let $\eta_{\varepsilon}(s)=W_{\varepsilon, s t_{0}}, s \in[0,1]$, then $\eta_{\varepsilon}(s) \in \Phi_{\varepsilon}$. Since $t_{0}>1$, from Lemma 3.1 we have

$$
\begin{aligned}
\limsup _{\varepsilon \rightarrow 0} C_{\varepsilon, \gamma, \pm} & \leqslant \limsup _{\varepsilon \rightarrow 0} \max _{s \in[0,1]} \Gamma_{\varepsilon, \gamma, \pm}\left(W_{\varepsilon, s t_{0}}\right) \\
& =\limsup _{\varepsilon \rightarrow 0} \max _{t \in\left[0, t_{0}\right]} \Gamma_{\varepsilon, \gamma, \pm}\left(W_{\varepsilon, t}\right) \\
& \leqslant \max _{t \in\left[0, t_{0}\right]} L_{m}\left(U_{t}\right)=C_{m}
\end{aligned}
$$

The result follows if we prove that $\liminf _{\varepsilon \rightarrow 0} C_{\varepsilon, \gamma, \pm} \geqslant C_{m}$. By the definition of $C_{\varepsilon, \gamma, \pm}$, for any $\widetilde{\varepsilon}>0$, there exists $\tilde{\eta}_{\varepsilon} \in \Phi_{\varepsilon}$ such that

$$
\max _{s \in[0,1]} \Gamma_{\varepsilon, \gamma, \pm}\left(\tilde{\eta}_{\varepsilon}(s)\right)<C_{\varepsilon, \gamma, \pm}+\widetilde{\varepsilon}
$$

Since $P_{\varepsilon, \gamma, \pm}\left(\tilde{\eta}_{\varepsilon}(0)\right)=0$ and $P_{\varepsilon, \gamma, \pm}\left(\tilde{\eta}_{\varepsilon}(1)\right) \leqslant \Gamma_{\varepsilon, \gamma, \pm}\left(\eta_{\varepsilon}(1)\right)=\Gamma_{\varepsilon, \gamma,+}\left(W_{\varepsilon, t_{0}}\right)<-1$, there exists $s_{0} \in(0,1)$ such that

$$
P_{\varepsilon, \gamma, \pm}\left(\tilde{\eta}_{\varepsilon}\left(s_{0}\right)\right)=-1 \quad \text { and } \quad P_{\varepsilon, \gamma, \pm}\left(\tilde{\eta}_{\varepsilon}(s)\right) \geqslant-1, \quad s \in\left[0, s_{0}\right) .
$$

Then we have

$$
Q_{\varepsilon}\left(\tilde{\eta}_{\varepsilon}(s)\right) \leqslant \Gamma_{\varepsilon, \gamma, \pm}\left(\tilde{\eta}_{\varepsilon}(s)\right)+1<C_{\varepsilon, \gamma, \pm}+\widetilde{\varepsilon}+1, \quad s \in\left[0, s_{0}\right] .
$$

By Lemma 2.2-(iii) we also have

$$
\begin{aligned}
\int_{\mathbb{R}^{N} \backslash \mathcal{O}_{\varepsilon}}\left|G_{\varepsilon, \gamma, \pm}^{-1}\left(\tilde{\eta}_{\varepsilon}(s)\right)\right|^{2} d x & \leqslant C \int_{\mathbb{R}^{N} \backslash \mathcal{O}_{\varepsilon}}\left|\tilde{\eta}_{\varepsilon}(s)\right|^{2} d x \\
& \leqslant C \varepsilon\left[\sqrt{Q_{\varepsilon}\left(\tilde{\eta}_{\varepsilon}(s)\right)}+1\right] \\
& \leqslant C \varepsilon\left[\sqrt{C_{\varepsilon, \gamma, \pm}+\widetilde{\varepsilon}+1}+1\right], \quad s \in\left[0, s_{0}\right] .
\end{aligned}
$$

Let us estimate $P_{\varepsilon, \gamma,+}$ and $P_{\varepsilon, \gamma,-}$ separately:

(i) Since $g_{\varepsilon, \gamma,+}^{2}(t) \geqslant 1$, we get

$$
\begin{aligned}
& P_{\varepsilon, \gamma,+}\left(\tilde{\eta}_{\varepsilon}(s)\right) \\
& \geqslant L_{m}\left(G_{\varepsilon, \gamma,+}^{-1}\left(\tilde{\eta}_{\varepsilon}(s)\right)\right)-\frac{1}{2} m \int_{\mathbb{R}^{N} \backslash \mathcal{O}_{\varepsilon}}\left|G_{\varepsilon, \gamma,+}^{-1}\left(\tilde{\eta}_{\varepsilon}(s)\right)\right|^{2} d x \\
& \geqslant L_{m}\left(G_{\varepsilon, \gamma,+}^{-1}\left(\tilde{\eta}_{\varepsilon}(s)\right)-\frac{1}{2} m \varepsilon C\left[\sqrt{C_{\varepsilon, \gamma,+}+\widetilde{\varepsilon}+1}+1\right], \quad s \in\left[0, s_{0}\right],\right.
\end{aligned}
$$


which yields

$$
L_{m}\left(G_{\varepsilon, \gamma,+}^{-1}\left(\tilde{\eta}_{\varepsilon}\left(s_{0}\right)\right) \leqslant \frac{1}{2} m \varepsilon C\left[\sqrt{C_{\varepsilon, \gamma,+}+\widetilde{\varepsilon}+1}+1\right]-1<0,\right.
$$

for small $\varepsilon>0$.

Thus, $G_{\varepsilon, \gamma,+}^{-1}\left(\tilde{\eta}_{\varepsilon}\left(t s_{0}\right)\right) \in \Phi$ and $\max _{t \in[0,1]} L_{m}\left(G_{\varepsilon, \gamma,+}^{-1}\left(\tilde{\eta}_{\varepsilon}\left(t s_{0}\right)\right) \geqslant C_{m}\right.$. So, by (3.9) and (3.10), we get

$$
\begin{aligned}
C_{\varepsilon, \gamma,+}+\widetilde{\varepsilon} & >\max _{s \in\left[0, s_{0}\right]} \Gamma_{\varepsilon, \gamma,+}\left(\tilde{\eta}_{\varepsilon}(s)\right) \\
& \geqslant C_{m}-\frac{1}{2} m \varepsilon C\left[\sqrt{C_{\varepsilon, \gamma,+}+\widetilde{\varepsilon}+1}+1\right],
\end{aligned}
$$

which yields $\liminf _{\varepsilon \rightarrow 0} C_{\varepsilon, \gamma,+} \geqslant C_{m}$ since $\widetilde{\varepsilon}$ is arbitrary.

(ii) For simplicity, let $u_{s}=G_{\varepsilon, \gamma,-}^{-1}\left(\tilde{\eta}_{\varepsilon}(s)\right), \omega_{s}(x)=u_{s}\left(\sqrt{1-4 \sqrt{\varepsilon^{\gamma}}} x\right)$, to have

$$
\begin{aligned}
P_{\varepsilon, \gamma,-}\left(\tilde{\eta}_{\varepsilon}(s)\right) & \\
\geqslant & \frac{1}{2} \int_{\mathbb{R}^{N}}\left(g_{\varepsilon, \gamma,-}^{2}\left(u_{s}\right)\left|\nabla u_{s}\right|^{2}+m u_{s}^{2}\right) d x-\int_{\mathbb{R}^{N}} H\left(u_{s}\right) d x-\frac{1}{2} m \int_{\mathbb{R}^{N} \backslash \mathcal{O}_{\varepsilon}} u_{s}^{2} d x \\
\geqslant & \left(1-4 \sqrt{\varepsilon^{\gamma}}\right)\left[\frac{1}{2} \int_{\mathbb{R}^{N}}\left(\left|\nabla u_{s}\right|^{2}+\frac{1}{1-4 \sqrt{\varepsilon^{\gamma}}} m u_{s}^{2}\right) d x-\frac{1}{1-4 \sqrt{\varepsilon^{\gamma}}} \int_{\mathbb{R}^{N}} H\left(u_{s}\right) d x\right] \\
& -\frac{1}{2} m \int_{\mathbb{R}^{N} \backslash \mathcal{O}_{\varepsilon}} u_{s}^{2} d x \\
\geqslant & \left(1-4 \sqrt{\varepsilon^{\gamma}}\right)^{-\frac{N}{2}}\left[\int_{\mathbb{R}^{N}} \frac{1}{2}\left(\left|\nabla \omega_{s}\right|^{2}+m \omega_{s}^{2}\right) d x-\int_{\mathbb{R}^{N}} H\left(\omega_{s}\right) d x\right] \\
& -\frac{1}{2} m \int_{\mathbb{R}^{N} \backslash \mathcal{O}_{\varepsilon}} u_{s}^{2} d x \\
\geqslant & \left(1-4 \sqrt{\varepsilon^{\gamma}}\right)^{-\frac{N}{2}} L_{m}\left(\omega_{s}\right)-\frac{1}{2} m \varepsilon C\left(\sqrt{C_{\varepsilon, \gamma,-}+\widetilde{\varepsilon}+1}+1\right), \quad s \in\left[0, s_{0}\right] .
\end{aligned}
$$

Hence

$$
L_{m}\left(\omega_{s_{0}}\right) \leqslant\left(1-4 \sqrt{\varepsilon^{\gamma}}\right)^{\frac{N}{2}}\left[\frac{1}{2} m \varepsilon C\left(\sqrt{C_{\varepsilon, \gamma,+}+\widetilde{\varepsilon}+1}+1\right)-1\right]<0,
$$

which implies $\omega_{t s_{0}}(x) \in \Phi$ and $\max _{t \in[0,1]} L_{m}\left(\omega_{t s_{0}}\right) \geqslant C_{m}$. So, by (3.9) and (3.11), we get

$$
\begin{aligned}
C_{\varepsilon, \gamma,-}+\widetilde{\varepsilon} & >\max _{s \in\left[0, s_{0}\right]} \Gamma_{\varepsilon, \gamma,-}\left(\tilde{\eta}_{\varepsilon}(s)\right) \\
& \geqslant\left(1-4 \sqrt{\varepsilon^{\gamma}}\right)^{-\frac{N}{2}} C_{m}-\frac{1}{2} m \varepsilon C\left[\sqrt{C_{\varepsilon, \gamma,-}+\widetilde{\varepsilon}+1}+1\right],
\end{aligned}
$$

which yields $\liminf _{\varepsilon \rightarrow 0} C_{\varepsilon, \gamma,-} \geqslant C_{m}$.

Combine (i) and (ii) to have $\liminf _{\varepsilon \rightarrow 0} C_{\varepsilon, \gamma, \pm} \geqslant C_{m}$ which completes the proof.

Remark 3.3. Let $D_{\varepsilon, \gamma, \pm}=\max _{s \in[0,1]} \Gamma_{\varepsilon, \gamma, \pm}\left(w_{\varepsilon, s t_{0}}\right)$; from Lemma 3.2 one has $\lim _{\varepsilon \rightarrow 0} D_{\varepsilon, \gamma, \pm}=$ $C_{m}$. 
Next we borrow some ideas from Byeon and Jeanjean [10] in order to prove the existence of critical points for the modified problem. This method has been used to deal with the case $\gamma=0$ in [16]. Here the situation is quite different as the limit equation is different from the case $\gamma=0$ and we set the problem in a Sobolev space setting instead of the Orlicz space setting used in [16].

We set the problem in the space $E_{\varepsilon}^{R}:=H_{0}^{1}\left(B_{R / \varepsilon}(0)\right)$ endowed with the norm

$$
\|v\|_{\varepsilon, R}=\left[\int_{B_{\frac{R}{\varepsilon}}(0)}\left(|\nabla v|^{2}+V_{\varepsilon}(x) v^{2}\right) d x\right]^{\frac{1}{2}} .
$$

Note that any $v \in E_{\varepsilon}^{R}$ can be regarded as an element in $E_{\varepsilon}$ by defining $v=0$ on $\mathbb{R}^{N} \backslash B_{\mathbb{R} / \varepsilon}(0)$, so that we may assume the two norms $\|\cdot\|_{\varepsilon}$ and $\|\cdot\|_{\varepsilon, R}$ coincide.

Define the level set

$$
\Gamma_{\varepsilon, \gamma, \pm}^{c}:=\left\{u \in E_{\varepsilon}^{R}: \Gamma_{\varepsilon, \gamma, \pm} \leqslant c\right\}
$$

and

$$
A^{d}:=\left\{u \in E_{\varepsilon}^{R}: \inf _{v \in A}\|u-v\|_{\varepsilon, R}<d\right\}, \quad d>0 .
$$

Let $v_{n} \in X_{\varepsilon_{n}}^{d} \cap E_{\varepsilon_{n}}^{R_{n}}$ with $\varepsilon_{n} \rightarrow 0$ and $R_{n} \rightarrow+\infty$ such that

$$
\lim _{n \rightarrow \infty} \Gamma_{\varepsilon_{n}, \gamma, \pm}\left(v_{n}\right) \leqslant C_{m} \text { and } \lim _{n \rightarrow \infty}\left\|\Gamma_{\varepsilon_{n}, \gamma, \pm}^{\prime}\left(v_{n}\right)\right\|_{\left(E_{\varepsilon_{n}}^{\left.R_{n}\right)^{\prime}}\right.}=0 .
$$

From the definition of $X_{\varepsilon_{n}}^{d}$, we can find $\left\{U_{n}\right\} \subset S_{m}$ and a sequence of points $\left\{y_{n}\right\} \subset \mathcal{M}^{\beta}$ such that

$$
\left\|v_{n}-\varphi_{\varepsilon_{n}}\left(\cdot-\frac{y_{n}}{\varepsilon_{n}}\right) U_{n}\left(\cdot-\frac{y_{n}}{\varepsilon_{n}}\right)\right\|_{\varepsilon_{n}, R_{n}} \leqslant d .
$$

Since $S_{m}$ and $\mathcal{M}^{\beta}$ are compact, there exist $U \in S_{m}$ and $y_{0} \in \mathcal{M}^{\beta}$ such that $U_{n} \rightarrow U$ in $H^{1}\left(\mathbb{R}^{N}\right), U_{n}(x) \rightarrow U(x)$ a.e. in $\mathbb{R}^{N}$ and $y_{n} \rightarrow y_{0}$. It is easy to check that for $d \in\left(0, d_{0}\right)$ with $d_{0}>0$ sufficiently small, we have

$$
\left\|v_{n}-\varphi_{\varepsilon_{n}}\left(\cdot-\frac{y_{n}}{\varepsilon_{n}}\right) U\left(\cdot-\frac{y_{n}}{\varepsilon_{n}}\right)\right\|_{\varepsilon_{n}, R_{n}} \leqslant 2 d,
$$

provided $n$ is large enough and in particular $\left\{v_{n}\right\}$ stays bounded in $E_{\varepsilon}$.

\section{Lemma 3.4.}

$$
\lim _{n \rightarrow \infty} \sup _{z \in\left\{z \in \mathbb{R}^{N}: \beta / 2 \leqslant\left|\varepsilon_{n} z-y_{n}\right| \leqslant 3 \beta\right\}} \int_{B_{R}(z)} v_{n}^{2} d x=0, \quad \forall R>0 .
$$

Proof. Suppose by contradiction that there exist $R>0$ and a sequence $\left\{z_{n}\right\} \subset\{z \in$ $\left.\mathbb{R}^{N}: \frac{1}{2} \beta \leqslant\left|\varepsilon_{n} z-y_{n}\right| \leqslant 3 \beta\right\}$ such that

$$
\lim _{n \rightarrow \infty} \int_{B_{R}\left(z_{n}\right)} v_{n}^{2} d x>0 .
$$


Let $\varepsilon_{n} z_{n} \rightarrow z_{0} \in\left\{z \in \mathbb{R}^{N}: \frac{1}{2} \beta \leqslant\left|z-y_{0}\right| \leqslant 3 \beta\right\}$, as $n \rightarrow \infty$. Set $\tilde{v}_{n}:=v_{n}\left(\cdot+z_{n}\right)$ and, up to a subsequence, we may assume $\tilde{v}_{n} \rightarrow \tilde{v}$ in $H^{1}\left(\mathbb{R}^{N}\right)$ and $\tilde{v}_{n} \rightarrow \tilde{v}$ in $L_{l o c}^{p}\left(\mathbb{R}^{N}\right)$, $p \in\left[2,2^{*}\right)$. Then, (3.14) yields

$$
\int_{B_{R}(0)}|\tilde{v}|^{2} d x=\lim _{n \rightarrow \infty} \int_{B_{R}(0)} \tilde{v}_{n}^{2} d x>0,
$$

which implies $\tilde{v} \not \equiv 0$.

From $\lim _{n \rightarrow \infty}\left\|\Gamma_{\varepsilon_{n}, \gamma, \pm}^{\prime}\left(v_{n}\right)\right\|_{\left(E_{\varepsilon_{n}}^{R_{n}}\right)^{\prime}}=0$, we have $\left\langle\Gamma_{\varepsilon_{n}, \gamma, \pm}^{\prime}\left(v_{n}\right), \phi\right\rangle=o_{n}(1)\|\phi\|$ for any $\phi \in C_{0}^{\infty}\left(\mathbb{R}^{N}\right)$. Hence

$$
\begin{aligned}
\int_{\mathbb{R}^{N}} \nabla \tilde{v}_{n} \nabla \phi & d x \\
& +\int_{\mathbb{R}^{N}} V_{\varepsilon_{n}}\left(x+z_{n}\right) \frac{G_{\varepsilon_{n}, \gamma, \pm}^{-1}\left(\tilde{v}_{n}\right)}{g_{\varepsilon_{n}, \gamma, \pm}\left(G_{\varepsilon_{n}, \gamma, \pm}^{-1}\left(\tilde{v}_{n}\right)\right)} \phi-\frac{h\left(G_{\varepsilon_{n}, \gamma, \pm}^{-1}\left(\tilde{v}_{n}\right)\right)}{g_{\varepsilon_{n}, \gamma, \pm}\left(G_{\varepsilon_{n}, \gamma, \pm}^{-1}\left(\tilde{v}_{n}\right)\right)} \phi d x \\
& -4\left(\int_{\mathbb{R}^{N}} \chi_{\varepsilon_{n}} v_{n}^{2} d x-1\right)_{+} \int_{\mathbb{R}^{N}} \chi_{\varepsilon_{n}}\left(x+z_{n}\right) \tilde{v}_{n} \phi d x=o_{n}(1)\|\phi\| .
\end{aligned}
$$

Clearly,

$$
\lim _{n \rightarrow \infty} \int_{\mathbb{R}^{N}} \chi_{\varepsilon_{n}}\left(x+z_{n}\right) \tilde{v}_{n} \phi d x=0 .
$$

Now let us prove

$$
\lim _{n \rightarrow \infty} \int_{\mathbb{R}^{N}} V_{\varepsilon_{n}}\left(x+z_{n}\right) \frac{G_{\varepsilon_{n}, \gamma, \pm}^{-1}\left(\tilde{v}_{n}\right)}{g_{\varepsilon_{n}, \gamma, \pm}\left(G_{\varepsilon_{n}, \gamma, \pm}^{-1}\left(\tilde{v}_{n}\right)\right)} \phi d x=V\left(z_{0}\right) \int_{\mathbb{R}^{N}} \tilde{v} \phi d x
$$

and

$$
\lim _{n \rightarrow \infty} \int_{\mathbb{R}^{N}} \frac{h\left(G_{\varepsilon_{n}, \gamma, \pm}^{-1}\left(\tilde{v}_{n}\right)\right)}{g_{\varepsilon_{n}, \gamma, \pm}\left(G_{\varepsilon_{n}, \gamma, \pm}^{-1}\left(\tilde{v}_{n}\right)\right)} \phi d x=\int_{\mathbb{R}^{N}} h(\tilde{v}) \phi d x .
$$

Indeed, by Lemma 2.2-(iv), one has $G_{\varepsilon_{n}, \gamma, \pm}^{-1}\left(\tilde{v}_{n}\right)-\tilde{v}_{n} \rightarrow 0$, a.e. in $\mathbb{R}^{N}$. Since $\tilde{v}_{n} \rightarrow \tilde{v}$, a.e. in $\mathbb{R}^{N}$, we get $G_{\varepsilon_{n}, \gamma, \pm}^{-1}\left(\tilde{v}_{n}\right) \rightarrow \tilde{v}$, a.e. in $\mathbb{R}^{N}$. Moreover, $g_{\varepsilon_{n}, \gamma, \pm}\left(G_{\varepsilon_{n}, \gamma, \pm}^{-1}\left(\tilde{v}_{n}\right)\right) \rightarrow 1$, a.e. in $\mathbb{R}^{N}$. Thus, (3.17) and (3.18) are consequences of the Lebesgue dominated convergence theorem.

Combine (3.16)-(3.18) to obtain

$$
\int_{\mathbb{R}^{N}}\left[\nabla \tilde{v} \nabla \phi+V\left(z_{0}\right) \tilde{v} \phi-h(\tilde{v}) \phi\right] d x=0, \quad \forall \phi \in C_{0}^{\infty}\left(\mathbb{R}^{N}\right),
$$

so that $\tilde{v}$ turns out to be a nontrivial solution to the following equation,

$$
-\Delta \tilde{v}+V\left(z_{0}\right) \tilde{v}=h(\tilde{v}), \quad x \in \mathbb{R}^{N} .
$$

By the maximum principle $\tilde{v}>0$. From $V\left(z_{0}\right) \geqslant m$, we have $C_{V\left(z_{0}\right)} \geqslant C_{m}$.

Next choose $R>0$ sufficiently large and apply Pohozaev's identity to get, on the one hand,

$$
2 \lim _{n \rightarrow \infty} \int_{B_{R}\left(z_{n}\right)}\left|\nabla v_{n}\right|^{2} d x \geqslant \int_{\mathbb{R}^{N}}|\nabla \tilde{v}|^{2} d x=N L_{V\left(z_{0}\right)}(\tilde{v}) \geqslant N C_{V\left(z_{0}\right)} \geqslant N C_{m},
$$


on the other hand, for large $n$, it follows from (3.13) that

$$
\begin{aligned}
\int_{B_{R}\left(z_{n}\right)}\left|\nabla v_{n}\right|^{2} d x \leqslant & 4 d^{2}+2 \varepsilon_{n}^{2} \int_{B_{R}\left(z_{n}\right)}\left|\nabla \varphi_{\varepsilon_{n}}\left(x-\frac{y_{n}}{\varepsilon_{n}}\right) U\left(x-\frac{y_{n}}{\varepsilon_{n}}\right)\right|^{2} d x \\
& +2 \int_{B_{R}\left(z_{n}\right)}\left|\varphi_{\varepsilon_{n}}\left(x-\frac{y_{n}}{\varepsilon_{n}}\right) \nabla U\left(x-\frac{y_{n}}{\varepsilon_{n}}\right)\right|^{2} d x \\
\leqslant & d+C \varepsilon_{n}^{2}+C \int_{B_{R}(0)} \exp \left(-c\left|x+z_{n}-\frac{y_{n}}{\varepsilon_{n}}\right|\right) d x .
\end{aligned}
$$

Note that $\lim _{n \rightarrow \infty}\left|z_{n}-\frac{y_{n}}{\varepsilon_{n}}\right|=+\infty$ since $\frac{1}{2 \varepsilon_{n}} \beta \leqslant\left|z_{n}-\frac{y_{n}}{\varepsilon_{n}}\right| \leqslant \frac{3 \beta}{\varepsilon_{n}}$. Thus, for $n$ large, we get

$$
\frac{1}{2} N C_{m} \leqslant \lim _{n \rightarrow \infty} \int_{B_{R}\left(z_{n}\right)}\left|\nabla v_{n}\right|^{2} d x \leqslant 3 d,
$$

which is a contradiction for small $d>0$. This completes the proof of Lemma 3.4.

Next choose $\eta \in C_{0}^{\infty}\left(\mathbb{R}^{N}\right)$ such that $0 \leqslant \eta \leqslant 1, \eta(z)=1$ if $z \in\left\{z \in \mathbb{R}^{N}: \beta \leqslant|z| \leqslant\right.$ $2 \beta\}, \eta(z)=0$ if $z \in \mathbb{R}^{N} \backslash\left\{z \in \mathbb{R}^{N}: \frac{1}{2} \beta \leqslant|z| \leqslant 3 \beta\right\}$. Setting $\eta_{n}(z)=\eta\left(\varepsilon_{n} z-y_{n}\right) v_{n}$, clearly, $\eta_{n}$ is bounded in $H^{1}\left(\mathbb{R}^{N}\right)$. Thus, from Lemma 3.7 , we have

$$
\lim _{n \rightarrow \infty} \sup _{z \in \mathbb{R}^{N}} \int_{\mathbb{R}^{N}}\left|\eta_{n}\right|^{2} d x=0
$$

This fact, together with Lions' lemma [21], gives $\eta_{n} \rightarrow 0$ in $L^{p+1}\left(\mathbb{R}^{N}\right)$. So that we obtain

$$
\begin{aligned}
& \lim _{n \rightarrow \infty} \int_{\left\{x \in \mathbb{R}^{N}: \beta \leqslant\left|\varepsilon_{n} x-y_{n}\right| \leqslant 2 \beta\right\}}\left|v_{n}\right|^{p+1} d x \\
& =\lim _{n \rightarrow \infty} \int_{\left\{x \in \mathbb{R}^{N}: \beta \leqslant\left|\varepsilon_{n} x-y_{n}\right| \leqslant 2 \beta\right\}}\left|\eta_{n}\right|^{p+1} d x \\
& \leqslant \lim _{n \rightarrow \infty} \int_{\mathbb{R}^{N}}\left|\eta_{n}\right|^{p+1} d x=0 .
\end{aligned}
$$

Set $v_{n, 1}=\varphi_{\varepsilon_{n}}\left(\cdot-\frac{y_{n}}{\varepsilon_{n}}\right) v_{n}$ and $v_{n, 2}=v_{n}-v_{n, 1}$. Then, we can prove the following lemma.

Lemma 3.5. $\Gamma_{\varepsilon_{n}, \gamma, \pm}\left(v_{n}\right) \geqslant \Gamma_{\varepsilon_{n}, \gamma, \pm}\left(v_{n, 1}\right)+\Gamma_{\varepsilon_{n}, \gamma, \pm}\left(v_{n, 2}\right)+o_{n}(1)$.

Proof. Note that $\operatorname{supp}\left(v_{n, 1}\right) \subset\left\{z \in \mathbb{R}^{N}:\left|\varepsilon_{n} z-y_{n}\right| \leqslant 2 \beta\right\} \subset \mathcal{M}_{\varepsilon}^{3 \beta} \subset \mathcal{O}_{\varepsilon}$, thus, $Q_{\varepsilon_{n}}\left(v_{n, 1}\right)=0$ and $Q_{\varepsilon_{n}}\left(v_{n, 2}\right)=Q_{\varepsilon_{n}}\left(v_{n}\right)$. On the other hand, we note that $\operatorname{supp}\left(v_{n, 2}\right) \subset\left\{z \in \mathbb{R}^{N}:\left|\varepsilon_{n} z-y_{n}\right| \geqslant \beta\right\}$. Therefore, by the convexity of $\left|G_{\varepsilon_{n}, \gamma, \pm}^{-1}(t)\right|^{2}$ 
and since $G_{\varepsilon_{n}, \gamma, \pm}^{-1}(0)=0$, for large $n$, we get

$$
\begin{aligned}
& \Gamma_{\varepsilon_{n}, \gamma, \pm}\left(v_{n, 1}\right)+\Gamma_{\varepsilon_{n}, \gamma, \pm}\left(v_{n, 2}\right) \\
&=\Gamma_{\varepsilon_{n}, \gamma, \pm}\left(v_{n}\right)+\int_{\mathbb{R}^{N}} \varphi_{\varepsilon_{n}}\left(x-\frac{y_{n}}{\varepsilon_{n}}\right)\left[\varphi_{\varepsilon_{n}}\left(x-\frac{y_{n}}{\varepsilon_{n}}\right)-1\right]\left|\nabla v_{n}\right|^{2} d x \\
& \quad+\frac{1}{2} \int_{\mathbb{R}^{N}} V\left(\varepsilon_{n} x\right)\left[\left|G_{\varepsilon_{n}, \gamma, \pm}^{-1}\left(v_{n, 1}\right)\right|^{2}+\left|G_{\varepsilon_{n}, \gamma, \pm}^{-1}\left(v_{n, 2}\right)\right|^{2}-\left|G_{\varepsilon_{n}, \gamma, \pm}^{-1}\left(v_{n}\right)\right|^{2}\right] d x \\
& \quad+\int_{\mathbb{R}^{N}}\left[H\left(G_{\varepsilon_{n}, \gamma, \pm}^{-1}\left(v_{n}\right)\right)-H\left(G_{\varepsilon_{n}, \gamma, \pm}^{-1}\left(v_{n, 1}\right)\right)-H\left(G_{\varepsilon_{n}, \gamma, \pm}^{-1}\left(v_{n, 2}\right)\right)\right] d x+o_{n}(1) \\
& \leqslant \Gamma_{\varepsilon_{n}, \gamma, \pm}\left(v_{n}\right)+\int_{\mathbb{R}^{N}}\left[H\left(G_{\varepsilon_{n}, \gamma, \pm}^{-1}\left(v_{n}\right)\right)\right. \\
&\left.-H\left(G_{\varepsilon_{n}, \gamma, \pm}^{-1}\left(v_{n, 1}\right)\right)-H\left(G_{\varepsilon_{n}, \gamma, \pm}^{-1}\left(v_{n, 2}\right)\right)\right] d x+o_{n}(1) .
\end{aligned}
$$

By $\left(h_{1}\right)-\left(h_{2}\right)$, given any $\sigma>0$, there exists some $C_{\sigma}>0$ such that

$$
\begin{aligned}
& \int_{\mathbb{R}^{N}}\left[H\left(G_{\varepsilon_{n}, \gamma, \pm}^{-1}\left(v_{n}\right)\right)-H\left(G_{\varepsilon_{n}, \gamma, \pm}^{-1}\left(v_{n, 1}\right)\right)-H\left(G_{\varepsilon_{n}, \gamma, \pm}^{-1}\left(v_{n, 2}\right)\right)\right] d x \\
& \leqslant \sigma \int_{\mathbb{R}^{N}} v_{n}^{2} d x+C_{\sigma} \int_{\left\{x \in \mathbb{R}^{N}: \beta \leqslant\left|\varepsilon_{n} x-y_{n}\right| \leqslant 2 \beta\right\}}\left|v_{n}\right|^{p+1} d x .
\end{aligned}
$$

Since $\sigma$ is arbitrary, by (3.21), (3.22) and (3.20) we obtain the result.

Lemma 3.6. $\Gamma_{\varepsilon_{n}, \gamma, \pm}\left(v_{n, 2}\right)>0$.

Proof. From (3.13), we get

$$
\begin{aligned}
& \int_{\left\{x \in \mathbb{R}^{N}:\left|\varepsilon_{n} x-y_{n}\right| \geqslant 2 \beta\right\}}\left|\nabla v_{n, 2}\right|^{2} d x=\int_{\left\{x \in \mathbb{R}^{N}:\left|\varepsilon_{n} x-y_{n}\right| \geqslant 2 \beta\right\}}\left|\nabla v_{n}\right|^{2} d x \\
& =\int_{\left\{x \in \mathbb{R}^{N}:\left|\varepsilon_{n} x-y_{n}\right| \geqslant 2 \beta\right\}}\left|\nabla\left[v_{n}-\varphi_{\varepsilon_{n}}\left(\cdot-\frac{y_{n}}{\varepsilon_{n}}\right) U\left(\cdot-\frac{y_{n}}{\varepsilon_{n}}\right)\right]\right|^{2} d x \\
& \leqslant\left\|v_{n}-\varphi_{\varepsilon_{n}}\left(\cdot-\frac{y_{n}}{\varepsilon_{n}}\right) U\left(\cdot-\frac{y_{n}}{\varepsilon_{n}}\right)\right\|_{\varepsilon_{n}}^{2} \leqslant d^{2} .
\end{aligned}
$$

Similarly, by (3.13), we get

$$
\begin{aligned}
& \int_{\left\{x \in \mathbb{R}^{N}: \beta \leqslant\left|\varepsilon_{n} x-y_{n}\right| \leqslant 2 \beta\right\}}\left|\nabla v_{n, 2}\right|^{2} d x \\
& \leqslant C \int_{\left\{x \in \mathbb{R}^{N}: \beta \leqslant\left|\varepsilon_{n} x-y_{n}\right| \leqslant 2 \beta\right\}}\left(\left|\nabla v_{n}\right|^{2}+v_{n}^{2}\right) d x \\
& \leqslant C d^{2}+\|\left.\left.\varphi_{\varepsilon_{n}}\left(\cdot-\frac{y_{n}}{\varepsilon_{n}}\right) U\left(\cdot-\frac{y_{n}}{\varepsilon_{n}}\right)\right|_{\varepsilon_{n}}\right|_{\left\{x \in \mathbb{R}^{N}: \beta \leqslant\left|\varepsilon_{n} x-y_{n}\right| \leqslant 2 \beta\right\}} \\
& \leqslant C d^{2}+o_{n}(1) .
\end{aligned}
$$


Consequently, as $n$ is large enough, we have $\left\|\nabla v_{n, 2}\right\|_{2} \leqslant C d$. For small $d>0$, using $\left(h_{1}\right)-\left(h_{2}\right)$ again, we get

$$
\begin{aligned}
& \Gamma_{\varepsilon_{n}, \gamma, \pm}\left(v_{n, 2}\right) \\
& \geqslant \frac{1}{2} \int_{\mathbb{R}^{N}}\left|\nabla v_{n, 2}\right|^{2} d x+\frac{1}{4} \int_{\mathbb{R}^{N}} V_{\varepsilon_{n}}(x)\left|G_{\varepsilon_{n}, \gamma, \pm}^{-1}\left(v_{n, 2}\right)\right|^{2} d x-C \int_{\mathbb{R}^{N}}\left|v_{n, 2}\right|^{2^{*}} d x \\
& \geqslant \frac{1}{2} \int_{\mathbb{R}^{N}}\left|\nabla v_{n, 2}\right|^{2} d x+\frac{1}{4} \int_{\mathbb{R}^{N}} V_{\varepsilon_{n}}(x)\left|G_{\varepsilon_{n}, \gamma, \pm}^{-1}\left(v_{n, 2}\right)\right|^{2} d x-C\left(\int_{\mathbb{R}^{N}}\left|\nabla v_{n, 2}\right|^{2} d x\right)^{\frac{2^{*}}{2}} \\
& \geqslant \frac{1}{4} \int_{\mathbb{R}^{N}}\left|\nabla v_{n, 2}\right|^{2} d x+\frac{1}{4} \int_{\mathbb{R}^{N}} V_{\varepsilon_{n}}(x)\left|G_{\varepsilon_{n}, \gamma, \pm}^{-1}\left(v_{n, 2}\right)\right|^{2} d x>0 .
\end{aligned}
$$

This completes the proof of Lemma 3.6.

Lemma 3.7. For $d>0$ sufficiently small, there exist a sequence $\left\{z_{n}\right\} \subset \mathbb{R}^{N}, y_{0} \in \mathcal{M}$, and $U_{0} \in S_{m}$ satisfying, up to a subsequence, the following:

$$
\lim _{n \rightarrow \infty}\left|\varepsilon_{n} z_{n}-y_{0}\right|=0, \quad \lim _{n \rightarrow \infty}\left\|v_{n}-\varphi_{\varepsilon_{n}}\left(\cdot-z_{n}\right) U_{0}\left(\cdot-z_{n}\right)\right\|_{\varepsilon_{n}, R_{n}}=0 .
$$

Proof. Let $w_{n}:=v_{n, 1}\left(\cdot+\frac{y_{n}}{\varepsilon_{n}}\right)$. Then, by (3.13), we get $\left\{w_{n}\right\}$ is bounded in $H^{1}\left(\mathbb{R}^{N}\right)$. Thus, up to a subsequence if necessary, we may assume $w_{n} \rightarrow w$ in $H^{1}\left(\mathbb{R}^{N}\right), w_{n} \rightarrow w$ in $L_{l o c}^{p}\left(\mathbb{R}^{N}\right), p \in\left[2,2^{*}\right)$ and $w_{n} \rightarrow w$ a.e. in $\mathbb{R}^{N}$. From (3.13), for given $R>0$, when $n$ large, we get

$$
\begin{aligned}
4 d^{2} & \geqslant \int_{\left\{x \in \mathbb{R}^{N}:\left|\varepsilon_{n} x-y_{n}\right| \leqslant \beta\right\}} V_{\varepsilon_{n}}(x)\left|v_{n, 1}-\varphi_{\varepsilon_{n}}\left(x-\frac{y_{n}}{\varepsilon_{n}}\right) U\left(x-\frac{y_{n}}{\varepsilon_{n}}\right)\right|^{2} d x \\
& =\int_{\left\{x \in \mathbb{R}^{N}:\left|\varepsilon_{n} x\right| \leqslant \beta\right\}} V\left(\varepsilon_{n} x+y_{n}\right)\left|w_{n}-U(x)\right|^{2} d x \\
& \geqslant m \int_{B_{R}(0)}\left|w_{n}-U(x)\right|^{2} d x .
\end{aligned}
$$

Thus, we have

$$
\begin{aligned}
\int_{B_{R}(0)} w^{2} d x & =\lim _{n \rightarrow \infty} \int_{B_{R}(0)} w_{n}^{2} d x \\
& \geqslant \int_{B_{R}(0)}|U(x)|^{2} d x-\lim _{n \rightarrow \infty} \int_{B_{R}(0)}\left|w_{n}-U(x)\right|^{2} d x \\
& \geqslant C-\frac{4}{m} d^{2},
\end{aligned}
$$

which yields $w \neq 0$.

Now let $\phi \in C_{0}^{\infty}\left(\mathbb{R}^{N}\right)$ and note that $w_{n}(x)=v_{n, 1}\left(x+\frac{y_{n}}{\varepsilon_{n}}\right)=v_{n}\left(x+\frac{y_{n}}{\varepsilon_{n}}\right)$ for $x \in \operatorname{supp}(\phi)$ and large $n$. Moreover, $\operatorname{supp}\left(w_{n}(x)\right) \subset\left\{x \in \mathbb{R}^{N}:\left|\varepsilon_{n} x\right| \leqslant 2 \beta\right\} \subset \mathcal{O}$. Thus, from $\left\langle\Gamma_{\varepsilon_{n}, \gamma, \pm}^{\prime}\left(v_{n}\right), \phi\right\rangle=o_{n}(1)\|\phi\|$ and analogous to the proof of (3.17) and (3.18), $w$ is a nontrivial solution of equation

$$
-\Delta w+V\left(y_{0}\right) w=h(w), \quad x \in \mathbb{R}^{N} .
$$

By the maximum principle, $w>0$. 
Now we claim:

$$
\lim _{n \rightarrow \infty} \sup _{z \in \mathbb{R}^{N}} \int_{B_{1}(z)}\left|w_{n}-w\right|^{2} d x=0 .
$$

In fact, if by contradiction (3.27) does not occur, then there exists a sequence $\left\{z_{n}\right\} \subset \mathbb{R}^{N}$ with $\left|z_{n}\right| \rightarrow+\infty$ such that

$$
\lim _{n \rightarrow \infty} \int_{B_{1}\left(z_{n}\right)}\left|w_{n}-w\right|^{2} d x>0
$$

Thus, we have

$$
\lim _{n \rightarrow \infty} \int_{B_{1}\left(z_{n}\right)}|w|^{2} d x=0, \quad \lim _{n \rightarrow \infty} \int_{B_{1}\left(z_{n}\right)}\left|w_{n}\right|^{2} d x>0 .
$$

We have $\left|\varepsilon_{n} z_{n}-y_{n}\right| \leqslant \frac{1}{2} \beta$. In fact, if $\left|\varepsilon_{n} z_{n}-y_{n}\right| \geqslant \frac{1}{2} \beta$, by (3.14), we have

$$
0<\lim _{n \rightarrow \infty} \int_{B_{1}\left(z_{n}\right)}\left|w_{n}\right|^{2} d x \leqslant \lim _{n \rightarrow \infty} \sup _{z \in\left\{z \in \mathbb{R}^{N}: \frac{1}{2} \beta \leqslant\left|\varepsilon_{n} z-y_{n}\right| \leqslant 3 \beta\right\}} \int_{B_{1}(z)}\left|v_{n}\right|^{2} d x=0,
$$

which is impossible. Thus, up to a subsequence, we may assume that $\varepsilon_{n} z_{n} \rightarrow z_{0} \in$ $\left\{z \in \mathbb{R}^{N}:|z| \leqslant 2 \beta\right\}$. Assume that $v_{n, 1}\left(\cdot+z_{n}+\frac{y_{n}}{\varepsilon_{n}}\right) \rightarrow v_{1}$ in $H^{1}\left(\mathbb{R}^{N}\right)$. Analogously to the proof of $(3.26)$, we have

$$
-\Delta v_{1}+V\left(z_{0}+y_{0}\right) v_{1}=h\left(v_{1}\right), \quad x \in \mathbb{R}^{N} .
$$

By the maximum principle, $v_{1}>0$.

Thus, for large $R$, we get

$$
\begin{aligned}
\frac{1}{2} N C_{m} & \leqslant \int_{B_{R}(0)}\left|\nabla v_{n, 1}\left(x+z_{n}+\frac{y_{n}}{\varepsilon_{n}}\right)\right|^{2} d x \\
& =\int_{B_{R}\left(z_{n}+\frac{y_{n}}{\varepsilon_{n}}\right)}\left|\nabla v_{n, 1}(x)\right|^{2} d x \\
& \leqslant C \varepsilon_{n}^{2}+C \int_{B_{R}\left(z_{n}+\frac{y_{n}}{\varepsilon_{n}}\right)}\left|\nabla v_{n}\right|^{2} d x \\
& \leqslant C \varepsilon_{n}^{2}+C d+C \int_{B_{R}(0)} e^{-c\left|x+z_{n}\right|} d x .
\end{aligned}
$$

We get a contradiction for large $n$ and small $d$ since $\left|z_{n}\right| \rightarrow+\infty$.

Therefore, (3.27) holds. By Lions' Lemma, we have $w_{n} \rightarrow w$ in $L^{p+1}\left(\mathbb{R}^{N}\right)$. Then, for given $\sigma>0$, there exists $C_{\sigma}>0$ such that

$$
\begin{aligned}
& \int_{\mathbb{R}^{N}}\left|H\left(G_{\varepsilon_{n}, \gamma, \pm}^{-1}\left(w_{n}\right)\right)-H\left(G_{\varepsilon_{n}, \gamma, \pm}^{-1}(w)\right)\right| d x \\
& =\int_{\mathbb{R}^{N}}\left|\frac{h\left(G_{\varepsilon_{n}, \gamma, \pm}^{-1}(\varpi)\right)}{g_{\varepsilon_{n}, \gamma, \pm}\left(G_{\varepsilon_{n}, \gamma, \pm}^{-1}(\varpi)\right)}\right|\left|w_{n}-w\right| d x \\
& \leqslant \sigma\left\|w_{n}-w\right\|_{2}^{2}+C_{\sigma}\left\|w_{n}-w\right\|_{p+1}^{p+1},
\end{aligned}
$$


where $\varpi(x)=t w_{n}(x)+(1-t) w(x), t \in(0,1)$. Thus, by the Lebesgue dominated convergence theorem,

$$
\lim _{n \rightarrow \infty} \int_{\mathbb{R}^{N}} H\left(G_{\varepsilon_{n}, \gamma, \pm}^{-1}\left(w_{n}\right)\right) d x=\int_{\mathbb{R}^{N}} H(w) d x .
$$

By Fatou's Lemma, Lemmas 3.5, 3.6 and (3.25), up to a subsequence, we have

$$
\begin{aligned}
C_{m} \geqslant & \lim _{n \rightarrow \infty} \Gamma_{\varepsilon_{n}, \gamma, \pm}\left(v_{n}\right) \\
\geqslant & \lim _{n \rightarrow \infty} \Gamma_{\varepsilon_{n}, \gamma, \pm}\left(v_{n, 1}\right)+\lim _{n \rightarrow \infty} \Gamma_{\varepsilon_{n}, \gamma, \pm}\left(v_{n, 2}\right) \\
\geqslant & \lim _{n \rightarrow \infty} \Gamma_{\varepsilon_{n}, \gamma, \pm}\left(v_{n, 1}\right) \\
= & \lim _{n \rightarrow \infty}\left[\frac{1}{2} \int_{\mathbb{R}^{N}}\left(\left|\nabla w_{n}\right|^{2}+V\left(\varepsilon_{n} x+y_{n}\right)\left|G_{\varepsilon_{n}, \gamma, \pm}^{-1}\left(w_{n}\right)\right|^{2}\right) d x\right. \\
& \left.-\int_{\mathbb{R}^{N}} H\left(G_{\varepsilon_{n}, \gamma, \pm}^{-1}\left(w_{n}\right)\right) d x\right] \\
\geqslant & \frac{1}{2} \int_{\mathbb{R}^{N}}\left(|\nabla w|^{2}+V\left(y_{0}\right) w^{2}\right) d x-\int_{\mathbb{R}^{N}} H(w) d x \\
\geqslant & C_{V\left(y_{0}\right)} \\
\geqslant & C_{m} .
\end{aligned}
$$

Hence, we get $\lim _{n \rightarrow \infty} \Gamma_{\varepsilon_{n}, \gamma, \pm}\left(v_{n, 1}\right)=C_{m}$. Moreover, we get $V\left(y_{0}\right)=m$ and as a consequence we see that $w$ is a ground state to (1.5). Thus, there exists some $z \in \mathbb{R}^{N}$ such that $U_{0}:=w(\cdot+z) \in S_{m}$. By (3.28), we have

$$
\begin{aligned}
& \lim _{n \rightarrow \infty}\left[\int_{\mathbb{R}^{N}}\left|\nabla w_{n}\right|^{2} d x+\int_{\mathbb{R}^{N}} V\left(\varepsilon_{n} x+y_{n}\right)\left|G_{\varepsilon_{n}, \gamma, \pm}^{-1}\left(w_{n}\right)\right|^{2} d x\right] \\
& =\left[\int_{\mathbb{R}^{N}}|\nabla w|^{2} d x+m \int_{\mathbb{R}^{N}} w^{2} d x\right] .
\end{aligned}
$$

This together Fatou's Lemma again give

$$
\lim _{n \rightarrow \infty} \int_{\mathbb{R}^{N}}\left|\nabla w_{n}\right|^{2} d x=\int_{\mathbb{R}^{N}}|\nabla w|^{2} d x
$$

and

$$
\lim _{n \rightarrow \infty} \int_{\mathbb{R}^{N}} V\left(\varepsilon_{n} x+y_{n}\right)\left|G_{\varepsilon_{n}, \gamma, \pm}^{-1}\left(w_{n}\right)\right|^{2} d x=m \int_{\mathbb{R}^{N}} w^{2} d x .
$$

Moreover, for any set $A \subset \mathbb{R}^{N}$, we get

$$
\lim _{n \rightarrow \infty} \int_{A} V\left(\varepsilon_{n} x+y_{n}\right)\left|G_{\varepsilon_{n}, \gamma, \pm}^{-1}\left(w_{n}\right)\right|^{2} d x=m \int_{A} w^{2} d x .
$$


By (3.29), we have

$$
\begin{aligned}
& \lim _{n \rightarrow \infty}\left\|\nabla\left[v_{n, 1}-\varphi_{\varepsilon_{n}}\left(\cdot-z-\frac{y_{n}}{\varepsilon_{n}}\right) w\left(\cdot-\frac{y_{n}}{\varepsilon_{n}}\right)\right]\right\|_{2} \\
& =\lim _{n \rightarrow \infty}\left\|\nabla\left[w_{n}-\varphi_{\varepsilon_{n}}(\cdot-z) w\right]\right\|_{2} \\
& \leqslant \lim _{n \rightarrow \infty}\left\|\nabla\left(w_{n}-w\right)\right\|_{2}+\lim _{n \rightarrow \infty}\left\|\nabla\left[\left(1-\varphi_{\varepsilon_{n}}(\cdot-z)\right) w\right]\right\|_{2}=0 .
\end{aligned}
$$

According to (3.30) and (3.31), buying the line of Proposition 2.1-(3) in [24], for any $\sigma>0$, we can take a bounded set $A_{\sigma} \subset \mathbb{R}^{N}$ and $n_{0} \in \mathbb{N}$ such that $\left|G_{\varepsilon_{n}, \gamma, \pm}^{-1}(t)\right|^{2}$ is convex and for $n>n_{0}$,

$$
\int_{\mathbb{R}^{N} \backslash A_{\sigma}} V\left(\varepsilon_{n} x+y_{n}\right)\left[\left|G_{\varepsilon_{n}, \gamma, \pm}^{-1}\left(\varphi_{\varepsilon_{n}}(x-z) w\right)\right|^{2}+\left|G_{\varepsilon_{n}, \gamma, \pm}^{-1}\left(w_{n}\right)\right|^{2}\right] d x \leqslant \frac{1}{2} \sigma .
$$

Thus, we have

$$
\int_{\mathbb{R}^{N} \backslash A_{\sigma}} V\left(\varepsilon_{n} x+y_{n}\right)\left|G_{\varepsilon_{n}, \gamma, \pm}^{-1}\left(w_{n}-\varphi_{\varepsilon_{n}}(x-z) w\right)\right|^{2} d x \leqslant C \sigma, \quad n>n_{0} .
$$

Thus, for small $\delta>0$, we get

$$
\begin{aligned}
& \left.\int_{\mathbb{R}^{N} \backslash A_{\sigma}} V\left(\varepsilon_{n} x+y_{n}\right)|| G_{\varepsilon_{n}, \gamma, \pm}^{-1}\left(w_{n}-\varphi_{\varepsilon_{n}}(x-z) w\right)\right|^{2} \\
& \quad-\left|w_{n}-\varphi_{\varepsilon_{n}}(x-z) w\right|^{2} \mid d x \\
& \leqslant \int_{\left\{x \in \mathbb{R}^{N} \backslash A_{\sigma}: 0<\left|\left(w_{n}-w\right)(x)\right| \leqslant \delta\right\}} V\left(\varepsilon_{n} x+y_{n}\right)\left|w_{n}-\varphi_{\varepsilon_{n}}(x-z) w\right|^{2} \\
& \quad \cdot\left|\frac{\left|G_{\varepsilon_{n}, \gamma, \pm}^{-1}\left(w_{n}-\varphi_{\varepsilon_{n}}(x-z) w\right)\right|^{2}}{\left|w_{n}-\varphi_{\varepsilon_{n}}(x-z) w\right|^{2}}-1\right| d x \\
& \quad+C \int_{\left\{x \in \mathbb{R}^{N} \backslash A_{\sigma}: \delta \leqslant\left|\left(w_{n}-\varphi_{\varepsilon_{n}}(x-z) w\right)(x)\right|\right\}}\left|w_{n}-\varphi_{\varepsilon_{n}}(x-z) w\right|^{p+1} d x \\
& \leqslant C \sigma\left\|w_{n}-\varphi_{\varepsilon_{n}}(x-z) w\right\|_{2}^{2}+C\left\|w_{n}-\varphi_{\varepsilon_{n}}(x-z) w\right\|_{p+1}^{p+1} .
\end{aligned}
$$

On the other hand, since $w_{n} \rightarrow w$ in $L_{l o c}^{2}\left(\mathbb{R}^{N}\right)$, we have

$$
\lim _{n \rightarrow \infty} \int_{A_{\sigma}} V\left(\varepsilon_{n} x+y_{n}\right)\left|w_{n}-\varphi_{\varepsilon_{n}}(x-z) w\right|^{2} d x=0 .
$$

Combining (3.32) and (3.33), we have

$$
\lim _{n \rightarrow \infty} \int_{\mathbb{R}^{N}} V\left(\varepsilon_{n} x\right)\left[v_{n, 1}-\varphi_{\varepsilon_{n}}\left(x-z-\frac{y_{n}}{\varepsilon_{n}}\right) w\left(x-\frac{y_{n}}{\varepsilon_{n}}\right)\right]^{2} d x=0 .
$$

Let $z_{n}=z+\frac{y_{n}}{\varepsilon_{n}}$, then

$$
\left\|v_{n, 1}-\varphi_{\varepsilon_{n}}\left(\cdot-z_{n}\right) U_{0}\left(\cdot-z_{n}\right)\right\|_{\varepsilon_{n}, R_{n}} \rightarrow 0 .
$$

Finally, using (3.28) again,

$$
0=\lim _{n \rightarrow \infty} \Gamma_{\varepsilon_{n}, \gamma, \pm}\left(v_{n, 2}\right) \geqslant \frac{1}{4} \lim _{n \rightarrow \infty} \int_{\mathbb{R}^{N}}\left[\left|\nabla v_{n, 2}\right|^{2}+V\left(\varepsilon_{n} x\right)\left|G_{\varepsilon_{n}, \gamma, \pm}^{-1}\left(v_{n, 2}\right)\right|^{2}\right] d x
$$

which yields $\lim _{n \rightarrow \infty}\left\|v_{n, 2}\right\|_{\varepsilon_{n}, R_{n}}=0$ by Lemma 2.2-(iii). The result follows. 
Let $d \in\left(0, d_{0}\right)$ such that Lemma 3.7 holds.

Lemma 3.8. For any $d \in\left(0, d_{0}\right)$, there exist positive constants $\delta_{d}, R_{d}$ and $\varepsilon_{d}$ such that

$$
\left\|\Gamma_{\varepsilon, \gamma, \pm}^{\prime}(v)\right\|_{\left(E_{\varepsilon}^{R}\right)^{\prime}} \geqslant \delta_{d}
$$

for any $v \in E_{\varepsilon}^{R} \cap \Gamma_{\varepsilon, \gamma, \pm}^{D_{\varepsilon, \gamma, \pm}} \cap\left(X_{\varepsilon}^{d_{0}} \backslash X_{\varepsilon}^{d}\right), R \geqslant R_{d}$, and $\varepsilon \in\left(0, \varepsilon_{d}\right)$.

Proof. By contradiction, we assume that for some $d \in\left(0, d_{0}\right)$, there exists $\varepsilon_{n}<\frac{1}{n}$, $R_{n}>n$ and $v_{n} \in E_{\varepsilon_{n}}^{R_{n}} \cap \Gamma_{\varepsilon_{n}, \gamma, \pm}^{D_{\varepsilon, \gamma}, \pm} \cap\left(X_{\varepsilon_{n}}^{d_{0}} \backslash X_{\varepsilon_{n}}^{d}\right)$ such that $\left\|\Gamma_{\varepsilon_{n}, \gamma, \pm}^{\prime}\left(v_{n}\right)\right\|_{\left(E_{\varepsilon_{n}}^{R_{n}}\right)^{\prime}}<\frac{1}{n}$. By

Lemma 3.7, there exist a sequence $\left\{z_{n}\right\} \subset \mathbb{R}^{N}, y_{0} \in \mathcal{M}$ and $U_{0} \in S_{m}$ satisfying

$$
\lim _{n \rightarrow \infty}\left|\varepsilon_{n} z_{n}-y_{0}\right|=0, \quad \lim _{n \rightarrow \infty}\left\|v_{n}-\varphi_{\varepsilon_{n}}\left(\cdot-z_{n}\right) U_{0}\left(\cdot-z_{n}\right)\right\|_{\varepsilon_{n}, R_{n}}=0
$$

up to a subsequence. Thus, for large $n, \varepsilon_{n} z_{n} \in \mathcal{M}^{\beta}, \varphi_{\varepsilon_{n}}\left(\cdot-z_{n}\right) U_{0}\left(\cdot-z_{n}\right) \in X_{\varepsilon_{n}}$ and $v_{n} \in X_{\varepsilon_{n}}^{d}$, which contradicts the fact $v_{n} \in X_{\varepsilon_{n}}^{d_{0}} \backslash X_{\varepsilon_{n}}^{d}$.

Lemma 3.9. For any given $\nu>0$, there exist small positive constants $\varepsilon_{1}$ and $d$ such that $\Gamma_{\varepsilon, \gamma, \pm}(v)>C_{m}-\nu$ for any $v \in X_{\varepsilon}^{d}$ and $\varepsilon \in\left(0, \varepsilon_{1}\right)$.

Proof. See [15].

Lemma 3.10. For sufficiently small $\varepsilon>0$ and large $R$, there exists a sequence $\left\{v_{\varepsilon, n}^{R}\right\} \subset E_{\varepsilon}^{R} \cap \Gamma_{\varepsilon, \gamma, \pm}^{D_{\varepsilon}, \gamma, \pm} \cap X_{\varepsilon}^{d_{0}}$ such that $\left\|\Gamma_{\varepsilon, \gamma, \pm}^{\prime}\left(v_{\varepsilon, n}^{R}\right)\right\|_{\left(E_{\varepsilon}^{R}\right)^{\prime}} \rightarrow 0$ as $n \rightarrow \infty$.

Proof. The proof follows $[15,16]$. However, for convenience the reader, we give a detailed proof. By contradiction, for small $\varepsilon>0$ and $R>R_{0}$, there exists $C(\varepsilon, R)>0$ such that

$$
\left\|\Gamma_{\varepsilon, \gamma, \pm}^{\prime}(v)\right\|_{\left(E_{\varepsilon}^{R}\right)^{\prime}} \geqslant C(\varepsilon, R), \quad v \in E_{\varepsilon}^{R} \cap \Gamma_{\varepsilon, \gamma, \pm}^{D_{\varepsilon}, \gamma, \pm} \cap X_{\varepsilon}^{d_{0}} .
$$

On the other hand, by Lemma 3.8, there exists $\delta>0$ independent of $\varepsilon \in\left(0, \varepsilon_{0}\right)$ and $R>R_{0}$ such that

$$
\left\|\Gamma_{\varepsilon, \gamma, \pm}^{\prime}(v)\right\|_{\left(E_{\varepsilon}^{R}\right)^{\prime}} \geqslant \delta, \quad v \in E_{\varepsilon}^{R} \cap \Gamma_{\varepsilon, \gamma, \pm}^{D_{\varepsilon}, \gamma, \pm} \cap\left(X_{\varepsilon}^{d_{0}} \backslash X_{\varepsilon}^{d_{1}}\right) .
$$

Thus, there exists a pseudo-gradient vector field $\Upsilon_{\varepsilon}^{R}$ on neighborhood $N_{\varepsilon}^{R} \subset E_{\varepsilon}^{R}$ of $E_{\varepsilon}^{R} \cap \Gamma_{\varepsilon, \gamma, \pm}^{D_{\varepsilon}, \gamma, \pm} \cap\left(X_{\varepsilon}^{d_{0}} \backslash X_{\varepsilon}^{d_{1}}\right)$. Let $\widetilde{N}_{\varepsilon}^{R} \subset N_{\varepsilon}^{R}$ such that

$$
\left\|\Gamma_{\varepsilon, \gamma, \pm}^{\prime}(v)\right\|_{\left(E_{\varepsilon}^{R}\right)^{\prime}} \geqslant \frac{1}{2} C(\varepsilon, R), \quad v \in \widetilde{N}_{\varepsilon}^{R} .
$$

We choose two positive Lipschitz continuous functions $\zeta_{\varepsilon}^{R}$ and $\xi$ satisfying

$$
\zeta_{\varepsilon}^{R}(v)=1, \quad \text { if } v \in \Gamma_{\varepsilon, \gamma, \pm}^{D_{\varepsilon}, \gamma, \pm} \cap X_{\varepsilon}^{d_{0}} \quad \text { and } \quad \zeta_{\varepsilon}^{R}(v)=0, \quad \text { if } v \in E_{\varepsilon}^{R} \backslash \tilde{N}_{\varepsilon}^{R},
$$

$0 \leqslant \zeta_{\varepsilon}^{R} \leqslant 1$, and

$$
\xi \leqslant 1, \quad \xi(a)=1, \quad \text { if }\left|a-C_{m}\right| \leqslant \frac{1}{2} C_{m} \quad \text { and } \quad \xi(a)=0, \quad \text { if }\left|a-C_{m}\right| \geqslant C_{m} .
$$

Now, we define

$$
\Psi_{\varepsilon}^{R}= \begin{cases}-\zeta_{\varepsilon}^{R}(v) \xi\left(\Gamma_{\varepsilon, \gamma, \pm}(v)\right) \Upsilon_{\varepsilon}^{R}, & \text { if } v \in N_{\varepsilon}^{R}, \\ 0, & \text { if } v \notin E_{\varepsilon} \backslash N_{\varepsilon}^{R} .\end{cases}
$$


Then the initial value problem

$$
\left\{\begin{array}{l}
\frac{d}{d t} F_{\varepsilon}^{R}(v, t)=\Psi_{\varepsilon}^{R}\left(F_{\varepsilon}^{R}(v, t)\right), \\
F_{\varepsilon}^{R}(v, 0)=v
\end{array}\right.
$$

yields a unique global solution $F_{\varepsilon}^{R}: E_{\varepsilon}^{R} \times \mathbb{R} \rightarrow E_{\varepsilon}^{R}$. For the properties of $F_{\varepsilon}^{R}$, we refer to [16]. Let $\eta_{\varepsilon}(s)=W_{\varepsilon, s t_{0}}=\varphi_{\varepsilon} U_{s t_{0}}, s \in[0,1]$ as before. Then, for the small $d_{1}>0$, there exists some $\mu>0$ such that if $\left|s t_{0}-1\right| \leqslant \mu$, we have

$$
\left\|\eta_{\varepsilon}(s)-\varphi_{\varepsilon} U\right\|_{\varepsilon}=\left\|\varphi_{\varepsilon}\left(U_{s t_{0}}-U\right)\right\|_{\varepsilon} \leqslant C\left\|U_{s t_{0}}-U\right\|_{\varepsilon} \leqslant d_{1},
$$

which implies that $\eta_{\varepsilon}(s) \in X_{\varepsilon}^{d_{1}}$ since $0 \in \mathcal{M}$. On the other hand, if $\left|s t_{0}-1\right| \geqslant \mu$, since $t=1$ is the unique maximum point of $L_{m}\left(U_{t}\right)$ and $\max _{t \geqslant 0} L_{m}\left(U_{t}\right)=L_{m}\left(U_{1}\right)=C_{m}$, there exists $\rho>0$ such that $L_{m}\left(U_{s t_{0}}\right)<C_{m}-2 \rho$ for $\left|s t_{0}-1\right| \geqslant \mu$. From Lemma 3.1 , there exists $\varepsilon_{1}>0$ such that

$$
\max _{s \in(0,1]}\left|\Gamma_{\varepsilon, \gamma, \pm}\left(W_{\varepsilon, s t_{0}}\right)-L_{m}\left(U_{s t_{0}}\right)\right|<\rho, \quad \varepsilon \in\left(0, \varepsilon_{1}\right) .
$$

So, for $\left|s t_{0}-1\right| \geqslant \mu$, we have

$$
\Gamma_{\varepsilon, \gamma, \pm}\left(W_{\varepsilon, s t_{0}}\right) \leqslant\left|\Gamma_{\varepsilon, \gamma, \pm}\left(W_{\varepsilon, s t_{0}}\right)-L_{m}\left(U_{s t_{0}}\right)\right|+L_{m}\left(U_{s t_{0}}\right)<C_{m}-\rho, \quad \varepsilon \in\left(0, \varepsilon_{1}\right) .
$$

Define $\eta_{\varepsilon}^{R}(s, t):=F_{\varepsilon}^{R}\left(\eta_{\varepsilon}(s), t\right),(s, t) \in[0,1] \times \mathbb{R}$. Since $\Gamma_{\varepsilon, \gamma, \pm}\left(\eta_{\varepsilon}(0)\right), \Gamma_{\varepsilon, \gamma, \pm}\left(\eta_{\varepsilon}(1)\right) \notin$ $\left(0,2 C_{m}\right)$, we get $\eta_{\varepsilon}^{R}(s, t) \in \Phi_{\varepsilon}$ for any $t>0$.

If $\left|s t_{0}-1\right| \geqslant \mu$, we have

$$
\Gamma_{\varepsilon, \gamma, \pm}\left(\eta_{\varepsilon}^{R}(s, t)\right) \leqslant \Gamma_{\varepsilon, \gamma, \pm}\left(\eta_{\varepsilon}(s)\right)<C_{m}-\rho,
$$

which is a contradiction.

If $\left|s t_{0}-1\right| \leqslant \mu$, we get $\eta_{\varepsilon}(s) \in X_{\varepsilon}^{d_{1}}$. In this case, one has (a) $\eta_{\varepsilon}^{R}(s, t) \in X_{\varepsilon}^{d_{0}}$ for all $t>0$ or (b) there exists some $t_{s}>0$ such that $\eta_{\varepsilon}^{R}(s, t) \notin X_{\varepsilon}^{d_{0}}$. If (a) holds, we have

$$
\begin{aligned}
\Gamma_{\varepsilon, \gamma, \pm}\left(\eta_{\varepsilon}^{R}(s, t)\right) & =\Gamma_{\varepsilon, \gamma, \pm}\left(\eta_{\varepsilon}(s)\right)+\int_{0}^{t} \frac{d}{d t} \Gamma_{\varepsilon, \gamma, \pm}\left(\eta_{\varepsilon}^{R}(s, \tau)\right) d \tau \\
& \leqslant D_{\varepsilon, \gamma, \pm}-\min \left\{\delta^{2}, C(\varepsilon, R)^{2}\right\} t .
\end{aligned}
$$

Thus, $\lim _{t \rightarrow+\infty} \Gamma_{\varepsilon, \gamma, \pm}\left(\eta_{\varepsilon}^{R}(s, t)\right)=-\infty$, which contradicts to Lemma 3.9. So, (b) holds. For any fixed $s$ with $\left|s t_{0}-1\right| \leqslant \mu$, we find $t_{s}^{1}, t_{s}^{2}>0$ such that $\eta_{\varepsilon}^{R}(s, t) \in X_{\varepsilon}^{d_{0}} \backslash \widetilde{X}_{\varepsilon}^{d_{1}}$ for $t \in\left[t_{s}^{1}, t_{s}^{2}\right] \subset\left(0, t_{s}\right)$ for $\left|t_{s}^{1}-t_{s}^{2}\right|>\sigma$ for some $\sigma>0$ dependent of $d_{0}$ and $d_{1}$. Thus,

$$
\begin{aligned}
\Gamma_{\varepsilon, \gamma, \pm}\left(\eta_{\varepsilon}^{R}\left(s, t_{s_{0}}\right)\right) & \leqslant \Gamma_{\varepsilon, \gamma, \pm}\left(\eta_{\varepsilon}(s)\right)+\int_{t_{s}^{1}}^{t_{s}^{2}} \frac{d}{d t} \Gamma_{\varepsilon, \gamma, \pm}\left(\eta_{\varepsilon}^{R}(s, \tau)\right) d \tau \\
& \leqslant D_{\varepsilon, \gamma, \pm}-\delta^{2}\left(t_{\varepsilon}^{2}-t_{\varepsilon}^{1}\right) \\
& \leqslant C_{m}-\frac{1}{2} \delta^{2} \sigma, \quad t \in\left[t_{s}^{1}, t_{s}^{2}\right], \quad \text { if }\left|s t_{0}-1\right| \leqslant \mu .
\end{aligned}
$$

Therefore, since $[0,1]$ is compact, using infinite covering theorem, for all $s \in[0,1]$, we can find $t_{\varepsilon}^{R}$ such that $\Gamma_{\varepsilon, \gamma, \pm}\left(\eta_{\varepsilon}^{R}\left(s, t_{\varepsilon}^{R}\right)\right)<C_{m}-\frac{1}{2} \delta^{2} d_{1}$. On the other hand, we 
note that $\eta_{\varepsilon}^{R}\left(s, t_{\varepsilon}^{R}\right) \in \Phi_{\varepsilon}$. Thus,

$$
C_{\varepsilon, \gamma, \pm} \leqslant \max _{s \in[0,1]} \Gamma_{\varepsilon, \gamma, \pm}\left(\eta_{\varepsilon}^{R}\left(s, t_{\varepsilon}^{R}\right)\right)<C_{m}-\rho,
$$

which is a contradiction.

Lemma 3.11. For sufficiently small $\varepsilon>0$, there exists a critical point $v_{\varepsilon} \in$ $X_{\varepsilon}^{d_{0}} \cap \Gamma_{\varepsilon, \gamma, \pm}^{D_{\varepsilon}, \gamma, \pm}$ of $\Gamma_{\varepsilon, \gamma, \pm}$.

Proof. By Lemma 8, there exists $\varepsilon_{0}$ and $R_{0}>0$ such that there exists a sequence $\left\{v_{\varepsilon, n}^{R}\right\} \subset E_{\varepsilon}^{R} \cap \Gamma_{\varepsilon, \gamma, \pm}^{D_{\varepsilon, \gamma} \pm} \cap X_{\varepsilon}^{d_{0}}$, such that $\left\|\Gamma_{\varepsilon, \gamma, \pm}^{\prime}\left(v_{\varepsilon, n}^{R}\right)\right\|_{\left(E_{\varepsilon}^{R}\right)^{\prime}} \rightarrow 0$ as $n \rightarrow \infty$ for $\varepsilon \in\left(0, \varepsilon_{0}\right)$, and $R \in\left(R_{0},+\infty\right)$. Clearly, $\left\{v_{\varepsilon, n}^{R}\right\}$ is bounded in $H_{0}^{1}\left(B_{\frac{R}{\varepsilon}}(0)\right)$ since $v_{\varepsilon, n}^{R} \in X_{\varepsilon}^{d_{0}}$. By defining $v_{\varepsilon, n}^{R}=0$ on $\mathbb{R}^{N} \backslash B_{\frac{R}{\varepsilon}}(0), v_{\varepsilon, n}^{R}$ can be regarded as an element in $E_{\varepsilon}$. Up to a subsequence if necessary, we may assume $v_{\varepsilon, n}^{R} \rightarrow v_{\varepsilon}^{R}$ in $H_{0}^{1}\left(B_{\frac{R}{\varepsilon}}(0)\right)$, $v_{\varepsilon, n}^{R} \rightarrow v_{\varepsilon}^{R}$ in $L^{p}\left(B_{\frac{R}{\varepsilon}}(0)\right), p \in\left[1,2^{*}\right)$ and $v_{\varepsilon, n}^{R} \rightarrow v_{\varepsilon}^{R}$ a.e. in $\mathbb{R}^{N}$. Thus $v_{\varepsilon}^{R}$ is a solution of

$$
-\Delta v=\frac{h\left(G_{\varepsilon, \gamma, \pm}^{-1}(v)\right)-V(\varepsilon x) G_{\varepsilon, \gamma, \pm}^{-1}(v)}{g_{\varepsilon, \gamma, \pm}\left(G_{\varepsilon, \gamma, \pm}^{-1}(v)\right)}-4\left(\int_{B_{\frac{R}{\varepsilon}(0)}} \chi_{\varepsilon}|v|^{2} d x-1\right) \chi_{\varepsilon} v, \quad x \in B_{\frac{R}{\varepsilon}}(0) .
$$

From (3.36) we have $v_{\varepsilon, n}^{R} \rightarrow v_{\varepsilon}^{R}$ in $H_{0}^{1}\left(B_{\frac{R}{\varepsilon}}(0)\right)$ and $v_{\varepsilon}^{R} \in X_{\varepsilon}^{d_{0}} \cap \Gamma_{\varepsilon, \gamma, \pm}^{D_{\varepsilon, \gamma}}$. By the maximum principle, $v_{\varepsilon}^{R}>0$. In consequence of $\left(h_{1}\right)-\left(h_{3}\right)$ and $\left|G_{\varepsilon, \gamma, \pm}^{-1}(t)\right| \leqslant \lambda_{2}|t|$ for all $t \in \mathbb{R}$, any positive solutions of (3.36) satisfies

$$
-\Delta v \leqslant C v^{p}, \quad x \in B_{\frac{R}{\varepsilon}}(0),
$$

where $C>0$ depends only on $h$. In particular, $-\Delta v_{\varepsilon}^{R} \leqslant C\left(v_{\varepsilon}^{R}\right)^{p}, x \in B_{\frac{R}{\varepsilon}}(0)$. By applying the standard Moser iteration argument (see [17]), $\left\{v_{\varepsilon}^{R}\right\}$ is bounded in $L_{l o c}^{p}\left(\mathbb{R}^{N}\right)$ uniformly on $R \geqslant R_{0}$ and $\varepsilon \in\left(0, \varepsilon_{0}\right)$ for any $p<\infty$ and is bounded in $L^{\infty}$. Thus, since $\left\|v_{\varepsilon}^{R}\right\|_{\varepsilon}$ and $\left\{\Gamma_{\varepsilon, \gamma, \pm}\left(v_{\varepsilon}^{R}\right)\right\}$ are bounded, we get $\left\{Q_{\varepsilon}\left(v_{\varepsilon}^{R}\right)\right\}$ is uniformly bounded on $R \geqslant R_{0}$ and $\varepsilon \in\left(0, \varepsilon_{0}\right)$. So, we have

$$
\int_{\mathbb{R}^{N} \backslash B_{\frac{R_{0}}{\varepsilon}}(0)}\left|v_{\varepsilon}^{R}\right|^{2} d x \leqslant \int_{\mathbb{R}^{N} \backslash \Omega_{\varepsilon}}\left|v_{\varepsilon}^{R}\right|^{2} d x=\varepsilon \int_{\mathbb{R}^{N}} \chi_{\varepsilon}\left|v_{\varepsilon}^{R}\right|^{2} d x \leqslant \varepsilon C
$$

for any $R \geqslant R_{0}$ and $\varepsilon \in\left(0, \varepsilon_{0}\right)$. Thus, for $|x| \geqslant \frac{R_{0}}{\varepsilon}$ and $R \geqslant R_{0}$, we have

$$
h\left(G_{\varepsilon, \gamma, \pm}^{-1}\left(v_{\varepsilon}^{R}\right)\right) \leqslant \frac{V_{0}}{2} G_{\varepsilon, \gamma, \pm}^{-1}\left(v_{\varepsilon}^{R}\right) .
$$

Similarly to Proposition 3 in [8], we get

$$
\lim _{A \rightarrow+\infty} \int_{\mathbb{R}^{N} \backslash B_{A}(0)}\left[\left|\nabla v_{\varepsilon}^{R}\right|^{2}+V_{\varepsilon}(x)\left|G_{\varepsilon}^{-1}\left(v_{\varepsilon}^{R}\right)\right|^{2}\right] d x=0
$$

uniformly on $R \geqslant R_{0}$. Let $v_{k}=v_{\varepsilon}^{R_{k}}$ and $R_{k} \rightarrow+\infty$ as $k \rightarrow \infty$. Then $\left\{v_{k}\right\}$ is bounded in $E_{\varepsilon}$ and we may assume that $v_{k} \rightarrow v_{\varepsilon}$ in $H^{1}\left(\mathbb{R}^{N}\right), v_{k} \rightarrow v_{\varepsilon}$ a.e. in $\mathbb{R}^{N}$. By iterating the above argument, we get $\left\|v_{\varepsilon}\right\|_{\infty} \leqslant C$. Since $v_{k}$ satisfies (3.36) and 
using (3.37), we get $\left\|v_{k}-v_{\varepsilon}\right\|_{\varepsilon} \rightarrow 0$ as $k \rightarrow \infty$. Thus, $v_{\varepsilon} \in X_{\varepsilon}^{d_{0}} \cap \Gamma_{\varepsilon, \gamma, \pm}^{D_{\varepsilon, \gamma, \pm}}$. Moreover, $\Gamma_{\varepsilon, \gamma, \pm}^{\prime}\left(v_{\varepsilon}\right)=0$.

\section{Proof of Theorem 1.1: the subcritical case}

By Lemma 3.11, for small $\varepsilon>0$, there exists a nonnegative solution $v_{\varepsilon}$ to

$$
-\Delta v+V_{\varepsilon}(x) \frac{G_{\varepsilon, \gamma, \pm}^{-1}(v)}{g_{\varepsilon, \gamma, \pm}\left(G_{\varepsilon, \gamma, \pm}^{-1}(v)\right)}=\frac{h\left(G_{\varepsilon, \gamma, \pm}^{-1}(v)\right)}{g_{\varepsilon, \gamma, \pm}\left(G_{\varepsilon, \gamma, \pm}^{-1}(v)\right)}-4\left(\int_{\mathbb{R}^{N}} \chi_{\varepsilon} v^{2} d x-1\right)_{+} \chi_{\varepsilon} v
$$

in $\mathbb{R}^{N}$. By the maximum principle we have $v_{\varepsilon}>0$. Since $v_{\varepsilon} \in X_{\varepsilon}^{d_{0}}$, by Moser's iteration and Lemma 2.2, $\left\{v_{\varepsilon}\right\}$ is uniformly bounded in $L^{\infty}\left(\mathbb{R}^{N}\right)$ for small $\varepsilon>0$. Moreover, there exists $\alpha>0$ such that $\left\|v_{\varepsilon}\right\|_{\infty} \geqslant \alpha$, see e.g. [35]. Thus, by choosing $\varepsilon>0$ sufficiently small, we get $g_{\varepsilon, \gamma, \pm}\left(G_{\varepsilon, \gamma, \pm}^{-1}\left(v_{\varepsilon}\right)\right)=1 \pm \varepsilon^{\gamma} G_{\varepsilon, \gamma, \pm}^{-1}\left(v_{\varepsilon}\right)^{2}$.

As in the proof of Lemma 3.7, there exist a sequence $\left\{z_{\varepsilon}\right\} \subset \mathbb{R}^{N}$ such that $\varepsilon z_{\varepsilon} \in \mathcal{M}^{2 \beta}$ and for any sequence $\varepsilon_{n} \rightarrow 0$ there exist $y_{0} \in \mathcal{M}$ and $U_{0} \in S_{m}$ satisfying

$$
\lim _{n \rightarrow \infty}\left|\varepsilon_{n} z_{\varepsilon_{n}}-y_{0}\right|=0, \quad \lim _{n \rightarrow \infty}\left\|v_{\varepsilon_{n}}-\varphi \varepsilon_{n}\left(\cdot-z_{\varepsilon_{n}}\right) U_{0}\left(\cdot-z_{\varepsilon_{n}}\right)\right\|_{\varepsilon_{n}}=0
$$

up to a subsequence. Thus, we get

$$
\lim _{n \rightarrow \infty}\left\|v_{\varepsilon_{n}}\left(\cdot+z_{\varepsilon_{n}}\right)-U_{0}\right\|_{H^{1}\left(\mathbb{R}^{N}\right)}=0
$$

which implies that for given $\sigma>0$, there exist $R>0$ and $\varepsilon_{0}>0$ such that

$$
\sup _{\varepsilon \in\left(0, \varepsilon_{0}\right)} \int_{\mathbb{R}^{N} \backslash B_{R}(0)} v_{\varepsilon_{n}}^{2}\left(x+z_{\varepsilon_{n}}\right) d x \leqslant \sigma .
$$

Setting $w_{\varepsilon}=v_{\varepsilon}\left(\cdot+z_{\varepsilon}\right)$, we have $-\Delta w_{\varepsilon} \leqslant M w_{\varepsilon}$. Hence, from [17], there exists a constant $C_{0}=C_{0}(N, C)$ such that

$$
\sup _{B_{1}(y)} w_{\varepsilon} \leqslant C_{0}\left\|w_{\varepsilon}\right\|_{L^{2}\left(B_{2}(y)\right)} \quad \text { for all } y \in \mathbb{R}^{N}
$$

Since $\left\|w_{\varepsilon}\right\|_{L^{2}\left(B_{2}(y)\right)} \rightarrow 0$, as $|y| \rightarrow \infty$, we conclude that $w_{\varepsilon}(x) \rightarrow 0$, as $|x| \rightarrow \infty$. Let $y_{\varepsilon}$ be a maximum point of $w_{\varepsilon}(x)$, then $\left\{y_{\varepsilon}\right\}$ is bounded. By the comparison principle we have

$$
w_{\varepsilon}(x) \leqslant C \exp (-c|x|) \quad \text { for all } \varepsilon \in\left(0, \varepsilon_{0}\right) .
$$

Hence, $x_{\varepsilon}:=y_{\varepsilon}+z_{\varepsilon}$ is a maximum point of $v_{\varepsilon}$ and

$$
v_{\varepsilon}(x)=w_{\varepsilon}\left(x-x_{\varepsilon}\right) \leqslant C \exp \left(-c\left|x-x_{\varepsilon}\right|\right) .
$$

Therefore, $Q_{\varepsilon}\left(v_{\varepsilon}\right)=0$ for small $\varepsilon>0$ and $v_{\varepsilon}$ is a positive critical point of $P_{\varepsilon, \gamma, \pm}$. Hence, $w_{\varepsilon, \gamma}=G_{\varepsilon, \gamma, \pm}^{-1}\left(v_{\varepsilon}\right)$ is a positive solution of (2.1). Let $y_{\varepsilon_{n}} \rightarrow y_{0}$ and $x=y_{0}+x_{0}$, then $\left\|v_{\varepsilon_{n}}\left(\cdot+x_{\varepsilon_{n}}\right)-U\left(\cdot+y_{0}\right)\right\|_{H^{1}} \rightarrow 0$. Furthermore,

$$
\left\|w_{\varepsilon_{n}, \gamma}\left(\cdot+x_{\varepsilon_{n}}\right)-U\left(\cdot+y_{0}\right)\right\|_{H^{1}}^{2} \leqslant C\left\|v_{\varepsilon_{n}}\left(\cdot+x_{\varepsilon_{n}}\right)-U\left(\cdot+y_{0}\right)\right\|_{H^{1}}^{2} \rightarrow 0 .
$$

The results follow by letting $u_{\varepsilon, \gamma}(x)=w_{\varepsilon, \gamma}\left(\frac{x}{\varepsilon}\right)$. 


\section{Proof of Theorem 1.1: the critical case}

In this Section, we deal with the existence and concentration of solutions to (1.1). We use a truncation approach, see also [37]. In the following, we first show the existence and qualitative properties of ground state solutions to the associated limit problem (1.5).

In [25], the authors proved that, if $\left(h_{1}\right)$ and $\left(h_{4}\right)-\left(h_{5}\right)$ hold, then there exists a ground state solution of (1.5) and the least energy $E_{m}$ is strictly less than $S^{N / 2} / N$, where $S$ is the Sobolev best constant, namely

$$
S=\inf _{u \in \mathcal{D}^{1,2}\left(\mathbb{R}^{N}\right) \backslash\{0\}} \frac{\|\nabla u\|_{2}^{2}}{\|u\|_{2^{*}}^{2}}
$$

Moreover, let

$$
\Upsilon(u)=\int_{\mathbb{R}^{N}}\left(H(u)-\frac{m}{2} u^{2}\right) \mathrm{d} x, u \in H^{1}\left(\mathbb{R}^{N}\right)
$$

and

$$
M:=\inf \left\{T(u): \Upsilon(u)=1, u \in H^{1}\left(\mathbb{R}^{N}\right)\right\} .
$$

Similarly to [38], we have

$$
E_{m}=\frac{2}{N} M^{\frac{N}{2}}\left(\frac{N-2}{N}\right)^{\frac{N-2}{2}}
$$

and then

$$
0<M<\frac{1}{2}\left(2^{*}\right)^{\frac{N-2}{N}} S .
$$

Let $S_{m}$ be the set of ground state solutions $U$ of (1.5) satisfying $U(0)=$ $\max _{x \in \mathbb{R}^{N}} U(x)$. Then $S_{m} \neq \emptyset$ and analogously to [9], the following result holds true:

\section{Proposition 5.1.}

(1) $S_{m}$ is compact in $H^{1}\left(\mathbb{R}^{N}\right)$;

(2) $0<\inf \left\{\|U\|_{\infty}: U \in S_{m}\right\} \leq \sup \left\{\|U\|_{\infty}: U \in S_{m}\right\}<\infty$.

By Proposition 5.1 there exists $\kappa>0$ such that

$$
\sup _{U \in S_{m}}\|U\|_{\infty}<\kappa \text {. }
$$

In the following, for any fixed $k>\max _{t \in[0, \kappa]} h(t)$, we consider the truncated problem

$$
-\Delta u+m u=h_{k}(u), \quad u \in H^{1}\left(\mathbb{R}^{N}\right),
$$

where

$$
h_{k}(t):=\min \{h(t), k\}, \quad t \in \mathbb{R} .
$$

Let $S_{m}^{k}$ be the set of positive ground state solutions $U$ of (5.3) satisfying $U(0)=$ $\max _{x \in \mathbb{R}^{N}} U(x)$. Then, similarly to [39], we have

\section{Lemma 5.2.}

$$
S_{m}^{k}=S_{m} \text { if } k>\max _{t \in[0, \kappa]} h(t) .
$$


Now we are in the position to prove the critical part of Theorem 1.1 by means of a truncation approach. Precisely, let us fix $k>\max _{t \in[0, \kappa]} f(t)$ and consider the truncated problem

$$
-\Delta w+V_{\varepsilon}(x) w \mp \varepsilon^{\gamma} w \Delta w^{2}=h_{k}(w), \quad x \in \mathbb{R}^{N},
$$

Similarly to Section 2 , by the change of variables $w=G_{\varepsilon, \gamma, \pm}^{-1}(v), v$ satisfies

$$
-\Delta v+V_{\varepsilon}(x) \frac{G_{\varepsilon, \gamma, \pm}^{-1}(v)}{g_{\varepsilon, \gamma, \pm}\left(G_{\varepsilon, \gamma, \pm}^{-1}(v)\right)}=\frac{h_{k}\left(G_{\varepsilon, \gamma, \pm}^{-1}(v)\right)}{g_{\varepsilon, \gamma, \pm}\left(G_{\varepsilon, \gamma, \pm}^{-1}(v)\right)}, \quad x \in \mathbb{R}^{N} .
$$

In view of Lemma $5.2, S_{m}^{k}=S_{m}$. For some fixed $\beta>0$ small and a cut-off function $\varphi \in C_{0}^{\infty}\left(\mathbb{R}^{N}\right)$ such that $0 \leq \varphi \leq 1, \varphi(x)=1$ for $|x| \leq \beta$ and $\varphi(x)=0$ for $|x| \geq 2 \beta$. Let $\varphi_{\varepsilon}(y)=\varphi(\varepsilon y), y \in \mathbb{R}^{N}$ and for each $x \in \mathcal{M}^{\beta}$ and $U \in S_{m}$, similarly as above, we define a set of approximating solutions by

$$
X_{\varepsilon}=\left\{U_{\varepsilon}^{x}(y) \mid x \in \mathcal{M}^{\beta}, U \in S_{m}\right\},
$$

where

$$
U_{\varepsilon}^{x}(y)=\varphi_{\varepsilon}\left(y-\frac{x}{\varepsilon}\right) U\left(y-\frac{x}{\varepsilon}\right) .
$$

Moreover, it follows from Theorem 1.1 that for some small $\varepsilon_{0}>0$ and $\varepsilon \in\left(0, \varepsilon_{0}\right)$, (5.5) admits a positive solution $v_{\varepsilon}$ satisfying the following property: there exist $U \in S_{m}$ and a maximum point $x_{\varepsilon} \in \mathbb{R}^{N}$ of $v_{\varepsilon}$, such that $\lim _{\varepsilon \rightarrow 0} \operatorname{dist}\left(x_{\varepsilon}, \mathcal{M}\right)=0$ and $v_{\varepsilon}\left(\varepsilon \cdot+x_{\varepsilon}\right) \rightarrow U\left(\cdot+z_{0}\right)$ as $\varepsilon \rightarrow 0$ in $H^{1}\left(\mathbb{R}^{N}\right)$ for some $z_{0} \in \mathbb{R}^{N}$. Clearly, $w_{\varepsilon}(\cdot)=v_{\varepsilon}\left(\varepsilon \cdot+x_{\varepsilon}\right)$ satisfies

$$
-\Delta w_{\varepsilon}+V_{\varepsilon}\left(x+\frac{x_{\varepsilon}}{\varepsilon}\right) \frac{G_{\varepsilon, \gamma, \pm}^{-1}\left(w_{\varepsilon}\right)}{g_{\varepsilon, \gamma, \pm}\left(G_{\varepsilon, \gamma, \pm}^{-1}\left(w_{\varepsilon}\right)\right)}=\frac{h_{k}\left(G_{\varepsilon, \gamma, \pm}^{-1}\left(w_{\varepsilon}\right)\right)}{g_{\varepsilon, \gamma, \pm}\left(G_{\varepsilon, \gamma, \pm}^{-1}\left(w_{\varepsilon}\right)\right)}, \quad x \in \mathbb{R}^{N} .
$$

Without loss of generality, we can choose $\varepsilon_{0}>0$ small enough such that $\frac{1}{2} \leq$ $g_{\varepsilon, \gamma, \pm}(t) \leq \frac{3}{2}$ uniformly for $t \in \mathbb{R}$ and $\varepsilon<\varepsilon_{0}$. Thanks to the fact that $h_{k}(t) \leq k$ for all $t \in \mathbb{R}$ and Lemma 2.2, in the week sense, $w_{\varepsilon}$ satisfies $-\Delta w_{\varepsilon}+\frac{2}{3} V_{0} w_{\varepsilon} \leq 2 k, x \in \mathbb{R}^{N}$. Then it follows from standard regularity estimates (see [17]) that $w_{\varepsilon}(\cdot) \rightarrow U\left(\cdot+z_{0}\right)$ in $C_{l o c}^{1, \alpha}\left(\mathbb{R}^{N}\right)$ as $\varepsilon \rightarrow 0$. In particular, $w_{\varepsilon}(0) \rightarrow U\left(z_{0}\right)$ as $\varepsilon \rightarrow 0$. Then it follows from (5.2) that $\left\|v_{\varepsilon}\right\|_{\infty}=w_{\varepsilon}(0)<\kappa$ holds for sufficiently small $\varepsilon>0$. Recalling that $0 \leq G_{\varepsilon, \gamma, \pm}^{-1}(t) \leq t$ for $t \geq 0$ and sufficiently small $\varepsilon>0$, we know $h_{k}\left(G_{\varepsilon, \gamma, \pm}^{-1}\left(v_{\varepsilon}(x)\right) \equiv h\left(G_{\varepsilon, \gamma, \pm}^{-1}\left(v_{\varepsilon}(x)\right), x \in \mathbb{R}^{N}\right.\right.$ for sufficiently small $\varepsilon>0$. Therefore, $v_{\varepsilon}$ turns out to be a positive solution of the original problem

$$
-\Delta v+V_{\varepsilon}(x) \frac{G_{\varepsilon, \gamma, \pm}^{-1}(v)}{g_{\varepsilon, \gamma, \pm}\left(G_{\varepsilon, \gamma, \pm}^{-1}(v)\right)}=\frac{h\left(G_{\varepsilon, \gamma, \pm}^{-1}(v)\right)}{g_{\varepsilon, \gamma, \pm}\left(G_{\varepsilon, \gamma, \pm}^{-1}(v)\right)}, \quad x \in \mathbb{R}^{N} .
$$

The proof of Theorem 1.1 is now complete.

Funding. Open access funding provided by Università degli Studi dell'Insubria within the CRUI-CARE Agreement. 
Open Access. This article is licensed under a Creative Commons Attribution 4.0 International License, which permits use, sharing, adaptation, distribution and reproduction in any medium or format, as long as you give appropriate credit to the original author(s) and the source, provide a link to the Creative Commons licence, and indicate if changes were made. The images or other third party material in this article are included in the article's Creative Commons licence, unless indicated otherwise in a credit line to the material. If material is not included in the article's Creative Commons licence and your intended use is not permitted by statutory regulation or exceeds the permitted use, you will need to obtain permission directly from the copyright holder. To view a copy of this licence, visit http://creativecommons.org/licenses/by/4.0/.

Publisher's Note. Springer Nature remains neutral with regard to jurisdictional claims in published maps and institutional affiliations.

\section{References}

[1] S. Adachi, T. Watanabe, Asymptotic properties of ground states of quasilinear Schrödinger equations with $H^{1}$-subcritical exponent, Adv. Nonlinear Stud., 12 (2012) 255-279. 2

[2] S. Adachi, M. Shibata, T. Watanabe, Blow-up phenomena and asymptotic profiles of ground states of quasilinear elliptic equations with $H^{1}$ supercritical nonlinearities, $J$. Differential Equations, 256 (2014) 1492-1514. 2

[3] S. Adachi, T. Watanabe, Asymptotic uniqueness of ground states for a class of quasilinear Schrödinger equations with $H^{1}$-supercritical exponents, J. Differential Equations, 260(3) (2016) 3086-3118. 2

[4] C.O. Alves, Y. Wang, Y. Shen, Soliton solutions for a class of quasilinear Schrödinger equations with a parameter, J. Differential Equations, 259(1) (2015) 318-343. 2, 3, 4

[5] H. Berestycki, P.-L. Lions, Nonlinear scalar field equations I, Arch. Rational Mech. Anal., 82 (1983), 313-346. 7

[6] L. Brizhik, A. Eremko, B. Piette, W.J. Zakrzewski, Electron self-trapping in a discrete two-dimensional lattice, Physica D., 159 (2001) 71-90. 2

[7] L. Brizhik, A. Eremko, B. Piette, W.J. Zakrzewski, Static solutions of a D-dimensional modified nonlinear Schrödinger equation, Nonlinearity, 16 (2003) 1481-1497. 2

[8] J. Byeon, Z. Wang, Standing waves with a critical frequency for nonlinear Schrödinger equations, II, Calc. Var., 18 (2003) 207-219. 7, 21

[9] J. Byeon, J. Zhang and W. Zou, Singularly perturbed nonlinear Dirichlet problems involving critical growth, Calc. Var. PDE., 47 (2013), 65-85. 3, 23

[10] J. Byeon, L. Jeanjean, Standing waves for nonlinear Schrödinger equations with a general nonlinearity, Arch. Rational Mech. Anal., 185(2007) 185-200. 3, 7, 11

[11] D. Cassani, J.M. do Ó, A. Moameni, Existence and concentration of solitary waves for a class of quasilinear Schrödinger equations, Commun. Pure Appl. Anal., 9(2) (2010) 281-306. 2 
[12] M. Colin, L. Jeanjean, Solutions for a quasilinear Schrödinger equations: A dual approach, Nonlinear Analysis TMA, 56 (2004), 213-226. 2, 3

[13] J.M. do Ó, U. Severo, Solitary waves for a class of quasilinear Schrödinger quations in dimension two, Calc. Var., 38(2010) 275-315. 2

[14] J.M. do Ó, A. Moameni, U. Severo, Semi-classical states for quasilinear Schrödinger equations arising in plasma physics, Commun. Cont. Math., 11(4) (2009) 547-583. 2

[15] E. Gloss, Existence and concentration of bound states for a p-Laplacian equation in $\mathbb{R}^{N}$, Adv. Nonlinear Stud., 10 (2010) 273-296. 19

[16] E. Gloss, Existence and concentration of positive solutions for a quasilinear equation in $\mathbb{R}^{N}$, J. Math. Anal. Appl., 371 (2010) 465-484. 2, 4, 11, 19, 20

[17] D. Gilbarg, N.S. Trudinger, Elliptic partical differential equation of second order, Springer, Berlin, 1989. 21, 22, 24

[18] X.M. He, A.X. Qian, W.M. Zou, Existence and concentration of positive solutions for quasilinear Schrödinger equations with critical growth, Nonlinearity, 26 (2013) 31373168. 2

[19] L. Jeanjean, K. Tanaka, A remark on the least enrgy solution in $\mathbb{R}^{N}$, Proc. Amer. Math. Soc., 131 (2003) 2399-2408. 7

[20] S. Kurihara, Large amplitude quasi-solitons in superfluid films, J. Phys. Soc. Japan., 50 (1981) 3262-3267. 2

[21] P.-L. Lions, The concentration-compactness principle in the calculus of variations. The locally compact case, part 1,2, Ann. Institute H. Poincaré, Analyses Nonlineaire, 1 (2\&3), 109-145, 223-283. 13

[22] J.Q. Liu, Z.Q. Wang, Soliton solutions for quasilinear Schrödinger equations, Proc. Amer. Math. Soc., 131 (2003) 441-448. 3

[23] J.Q. Liu, Y.Q. Wang, Z.Q. Wang, Soliton solutions to quasilinear Schrödinger equations II, J. Differential Equations, 187 (2003) 473-493. 2, 3

[24] J.Q. Liu, Y.Q. Wang, Z.Q. Wang, Solutions for quasilinear Schrödinge equations via the Nehari method, Comm. Partial Differential Equations, 29 (2004) 879-901. 2, 3, 18

[25] J. Liu, J. Liao and C.-L. Tang, Ground state solution for a class of Schrödinger equations involving general critical growth term, Nonlinearity, 30 (2017), 899-911. 23

[26] A. Nakamura, Damping and modification of exciton solitary waves, J. Phys. Soc. Japan, 42 (1977) 1824-1835. 2

[27] M. Poppenberg, K. Schmitt, Z.Q. Wang, On the existence of soliton solutions to quasilinear Schrödinger equations, Calc. Var. Partial Differential Equations, 14 (2002) 329-344. 2

[28] J. Sreekumar, V.M. Nandakumaran, Two dimensional large amplitude quasi solitons in thin helium films, Modern Physics Letters B, 4 (1990) 41-51. 2

[29] Y. Shen, Y. Wang, Soliton solutions for generalized quasilinear Schrödinger equations, Nonlinear Analysis TMA, 80 (2013), 194-201. 3, 6

[30] Y. Shen, Y. Wang, A class of generalized quasilinear Schrödinger equations, Comm. Pure Appl. Anal., 15 (2016), 853-870. 2

[31] W. Krolikowski, O. Bang, J.J. Rasmussen, J. Wyller, Modulational instability in nonlocal nonlinear Kerr media, Phys. Rev. E., 64 (2001) 0166122 
[32] U.B. Severo, E. Gloss, E.D. da Silva, On a class of quasilinear Schrödinger equations with superlinear or asymptotically linear terms, J. Differential Equations, 263 (2017) 3550-3580. 2, 3, 4

[33] Y. Wang, W. Zou, Bound states to critical quasilinear Schrödinger equations, Nonlinear differ. equ. appl., 19 (2012) 19-47. 2

[34] Y. Wang, Y. Shen, Existence and asymptotic behavior of a class of quasilinear Schrödinger equations, Advanced Nonlinear Studies, 18(1) (2018) 131-150. 2

[35] X.B. Pan, Blow-up behavior of ground states of semilinear elliptic Equations in $\mathbb{R}^{N}$ involving critical Sobolev exponents, J. Differential Equations, 99 (1992) 78-107. 22

[36] M.B. Yang, Y.H. Ding, Existence of semiclassical states for a quasilinear Schrödinger equation with critical exponent in $\mathbb{R}^{N}$, Annali di Matematica Pura ed Applicata, 192 (2013) 783-804. 2

[37] J. Zhang and J.M. do Ó, Standing waves for nonlinear Schrödinger equations involving critical growth of Trudinger-Moser type, Z. Angew. Math. Phys., 66(2015), 3049-3060. 23

[38] J. Zhang and W. Zou, A Berestycki-Lions Theorem revisited, Commun. Contemp. Math., 14 (2012), 1250033. 23

[39] J. Zhang, Standing waves with a critical frequency for nonlinear Schrödinger equations involving critical growth, Appl. Math. Lett., 63(2017), 53-58. 23

Daniele Cassani

Dip. di Scienza e Alta Tecnologia

Università degli Studi dell'Insubria

Via Valleggio 11, 22100 Como

Italy

and

RISM-Riemann International School of Mathematics

Villa Toeplitz, Via G.B. Vico 46, 21100 Varese

Italy

e-mail: Daniele.Cassani@uninsubria.it

Youjun Wang

Department of Mathematics

South China University of Technology

Guangzhou 510640

P.R. China

and

Dip. di Scienza e Alta Tecnologia

Università degli Studi dell'Insubria

Via Valleggio 11, 22100 Como

Italy

e-mail: scyjwang@scut.edu.cn 
Jianjun Zhang

College of Mathematics and Statistics

Chongqing Jiaotong University

Chongqing 400074

P.R. China

e-mail: zhangjianjun09@tsinghua.org.cn

Submitted: July 22, 2020.

Accepted: October 16, 2020. 